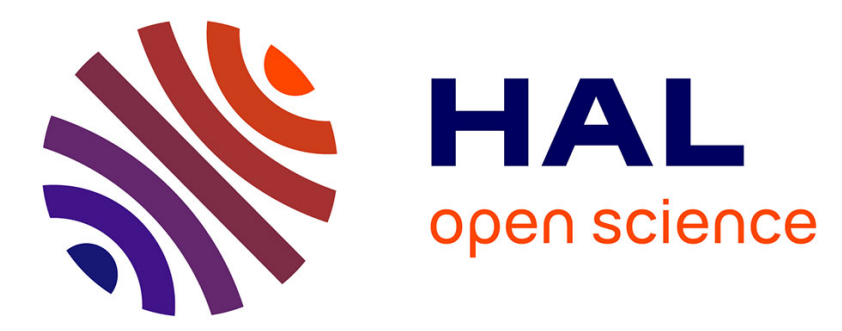

\title{
Vascular and extracellular matrix remodeling by physical approaches to improve drug delivery at the tumor site
} Sara Gouarderes, Anne-Françoise Mingotaud, Patricia Vicendo, Laure Gibot

\section{To cite this version:}

Sara Gouarderes, Anne-Françoise Mingotaud, Patricia Vicendo, Laure Gibot. Vascular and extracellular matrix remodeling by physical approaches to improve drug delivery at the tumor site. Expert Opinion on Drug Delivery, 2020, 17 (12), pp.1703-1726. 10.1080/17425247.2020.1814735 . hal-02927112

\section{HAL Id: hal-02927112 \\ https://hal.science/hal-02927112}

Submitted on 3 Sep 2020

HAL is a multi-disciplinary open access archive for the deposit and dissemination of scientific research documents, whether they are published or not. The documents may come from teaching and research institutions in France or abroad, or from public or private research centers.
L'archive ouverte pluridisciplinaire HAL, est destinée au dépôt et à la diffusion de documents scientifiques de niveau recherche, publiés ou non, émanant des établissements d'enseignement et de recherche français ou étrangers, des laboratoires publics ou privés. 
Sara Gouarderes ${ }^{1}$, Anne-Françoise Mingotaud ${ }^{1}$, Patricia Vicendo ${ }^{1}$, Laure Gibot ${ }^{1, *}$

4

1 Laboratoire des IMRCP, Université de Toulouse, CNRS UMR 5623, Université Toulouse III - Paul

5 Sabatier, France

6

*Author for correspondence: Laure Gibot, +335 615562 72, gibot@chimie.ups-tlse.fr

7

\section{Graphical abstract}

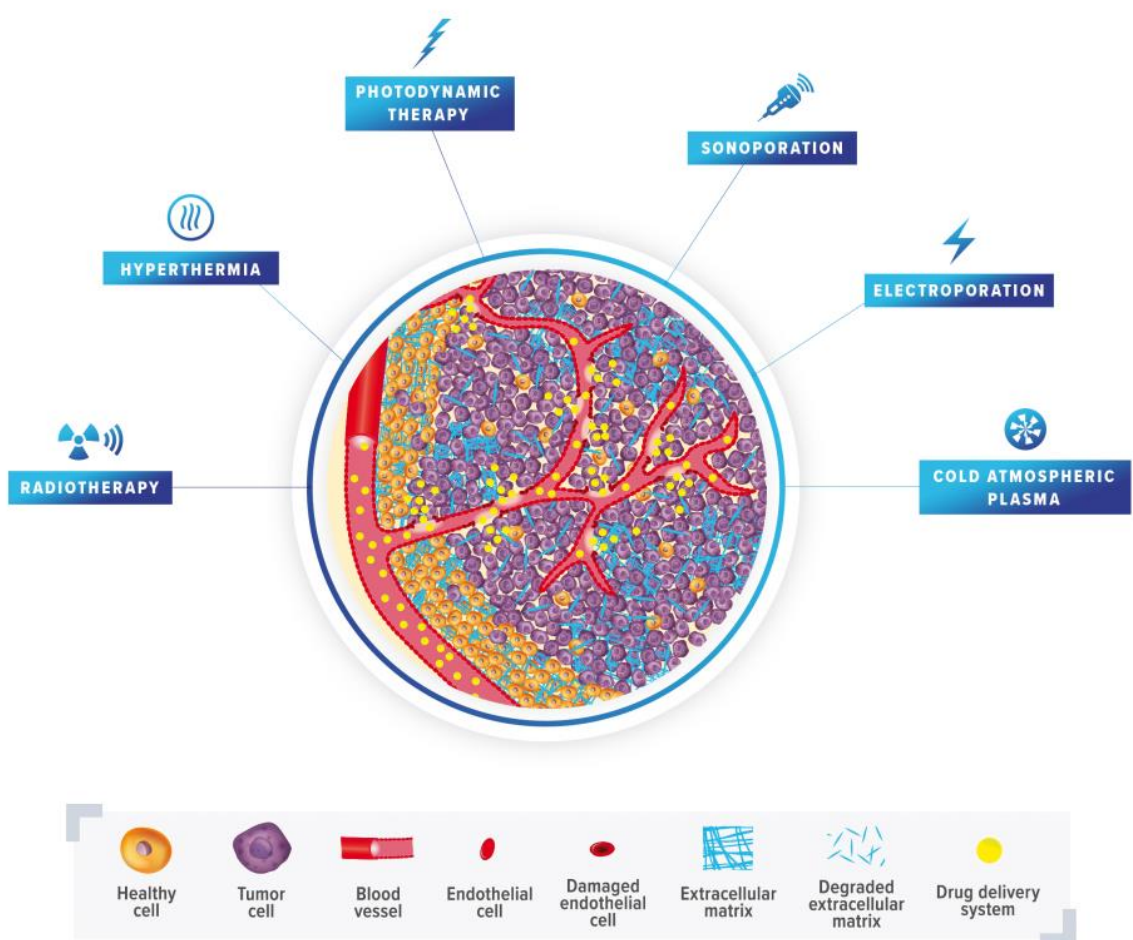

9

Abstract

Introduction: Modern comprehensive studies of tumour microenvironment changes allowed scientists to develop new and more efficient strategies that will improve anticancer drug delivery on site. The tumour microenvironment, especially the dense extracellular matrix, has a recognised capability to hamper the penetration of conventional drugs. Development and co-applications of strategies aiming at remodelling the tumour microenvironment are highly demanded to improve drug delivery at the 
tumour site in a therapeutic prospect. Areas covered: Increasing indications suggest that classical physical approaches such as exposure to ionising radiation, hyperthermia or light irradiation, and emerging ones as sonoporation, electric field or cold plasma technology can be applied as standalone or associated strategies to remodel the tumour microenvironment. The impacts on vasculature and extracellular matrix remodelling of these physical approaches will be discussed with the goal to improve nanotherapeutics delivery at the tumour site. Expert opinion: Physical approaches to modulate vascular properties and remodel the extracellular matrix are of particular interest to locally control and improve drug delivery and thus increase its therapeutic index. They are particularly powerful as adjuvant to nanomedicine delivery; the development of these technologies could have extremely widespread implications for cancer treatment.

\section{Key words}

Collagens, ECM, tumour microenvironment, radiotherapy, hyperthermia, photodynamic therapy (PDT), electroporation, sonoporation, cold atmospheric plasma, vascular permeability

\section{Article highlights}

- As opposed to pharmacological strategies, physical approaches allow a spatial and temporal treatment of the target tissue.

- Radiotherapy (RT), by damaging tumour vasculature and ECM, enhance nanotherapeutics drug delivery and subsequent increase of RT activity at the tumour site leading to significant reduction of both resistance processes and tumour hypoxia.

- Hyperthermia therapy, focused ultrasounds or alternative magnetic fields, are three approaches that prime the tumour by disrupting extracellular matrix and tumour vasculature, enhancing on-site drug delivery. 
- Chemophototherapy, the combination of phototherapy and chemotherapy, is an efficient strategy to induce tumour vascular permeability and ensuing drug delivery to tumours.

- Standalone or co-applied physical strategies to disrupt the dense tumour extracellular matrix or to mediate vascular permeability may be of major interest to improve drug delivery at the tumour site in a therapeutic prospect.

\section{Introduction}

In the field of oncology, intensive works carried out by researchers have highlighted many behaviours specific to cancer, so that invasive therapies are being replaced by approaches refined by the knowledge accumulated over the years. The tumour microenvironment (TME) plays a key role in the effective delivery of anti-cancer drugs on the tumour. Indeed, TME has a recognised capability to hamper the penetration of conventional drugs and drug delivery systems because of the tumour growth process itself $[1,2]$. To overcome that specific barrier to anti-cancer drugs, researchers have explored new challenging strategies they have adapted from a deep understanding of cancer growth process. Actually, to obtain nutrients supply to fuel their growth and to facilitate their dissemination to other organs, cancer cells promote the birth of new blood vessels from existing ones, through a process known as tumour angiogenesis. Tumour blood vessels are tortuous, dilated and leaky. These structural and functional defects [3] make the immature vascular walls within the tumour microenvironment more permeable, especially to macromolecules as proven in 1986 by Matsumura et al. They found that high molecular weight anticancer agents can passively accumulate in solid tumours due to the enhanced permeability and retention (EPR) effect [4] and non-functional lymphatics . This passive accumulation of drug delivery systems in tumour tissue increases the efficacy of the transported drugs. However, despite promising initial preclinical results of passively targeted chemotherapeutic drug delivery through the EPR effect, serious questions have been raised about the existence and clinical application of the EPR effect in human tumours [5]. Indeed, drug trafficking within a solid tumour is dramatically slowed down because of significant impediments such as high 
tumour interstitial fluid pressure related to defective lymphatic microvasculature [6], and chaotic microvasculature of most solid tumours. Besides, vascular permeability based on EPR effect may differ greatly between different types of tumours and even within one tumour. Thus, several pharmacological and physical strategies that can improve the EPR effect to facilitate nanomedicine delivery in viable tumour cells in relevant concentrations are of high importance and they have been assessed and nicely described in other reviews dedicated on these topics [7-11].

Another important physical barrier to drug and drug delivery systems is the densification of the extracellular matrix (ECM) in the tumour microenvironment (Figure 1) [12]. Indeed, the tumorous ECM is the most abundant component in tumour environment and it regulates cancer development by eliciting various biochemical and biophysical signalling [13]. For example, type I and III collagen production are enhanced and associated with the formation of aberrant collagen bundles during tumour progression [14]. Densification of tumour ECM renders it stiffer compared to the healthy matrix $[15,16]$. As a negative consequence, total collagen content negatively affects vascular macromolecule transport, possibly by binding and stabilising the glycosaminoglycan component of the ECM [17]. The interest in promoting the degradation of the extracellular matrix is double [18]. Firstly, the lower matrix density facilitates the diffusion of drugs and drug delivery systems within the tissue. Secondly, less matrix means lower interstitial fluid pressure (IFP) within the tumour, less compression of blood vessels and thus improved drug distribution.

Healthy tissue

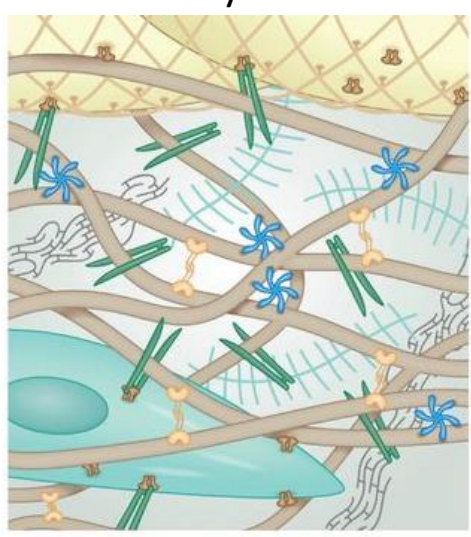

\section{Tumour tissue}

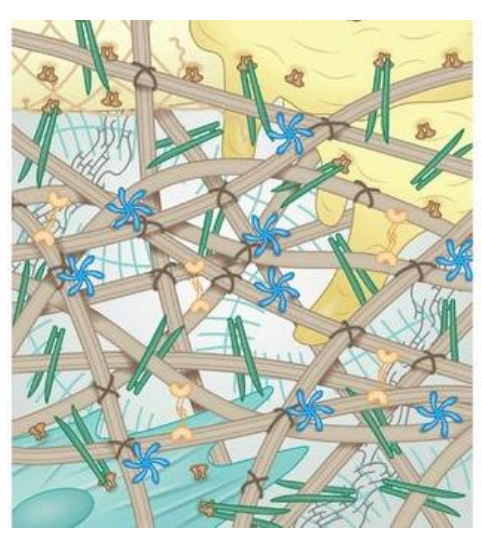

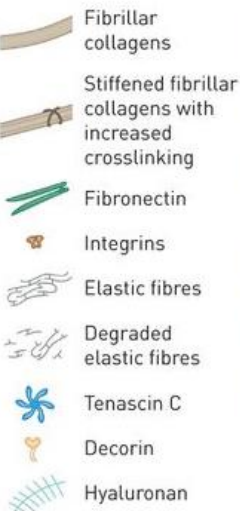

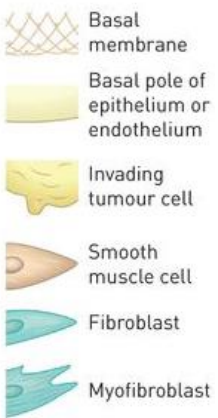

Myofibroblast

Figure 1: Changes observed within the extracellular matrix in the tumour tissue. In healthy tissue ECM 21 is remodelled by the activity of fibroblasts and presents a loose meshwork of collagen, elastin and 
fibronectin. In cancer, tumours are surrounded by a dense and stiff ECM containing highly crosslinked collagen, and high levels of fibronectin, tenascin $C$ and hyaluronan. Adapted from [19], reproduced with permission of the (C) ERS 2020: European Respiratory Journal 50 (1) 1601805; DOI: 10.1183/13993003.01805-2016 Published 5 July 2017.

In a nutshell, distances between blood vessels in tumour cells, composition and architecture of the extracellular matrix, intercellular junctions, high interstitial fluid pressure, lack of convection, drug metabolism and binding all contribute to various extents to limited drug distribution [20]. Consequently, any strategy enabling anticancer drugs and/or nanomedicine distribution improvement in tumours will thereby increase their therapeutic index.

Matrix remodelling is one of the most valuable approaches to improve the delivery of therapeutic agents as evidenced in vivo with single or chronic injection of pharmacological agents able to degrade tumour ECM proteins. Among them, let's cite hormones like relaxin [21], enzymes like collagenases [22], angiotensin inhibitor called losartan [23], the antibody simtuzumab to inhibit the lysyl oxidaselike 2 (LOXL2) [24]. Unfortunately, metalloproteinases (MMP) inhibitors (such as marimastat and prinomastat) have not been clinically proven to be effective against cancer, due to their lack of specificity and poor tolerability [25]. One of the flagship studies combining pharmacological ECM matrix remodelling and concomitant treatment with anticancer nanoparticles was published in 2010 by Jain's team. The authors showed that the repetitive injection of losartan decreased collagen amounts in several mouse tumour models, which in turn favour a higher penetration of doxorubicinloaded liposomes (Doxil) into the tumour, particularly in the interstitial space, thus improving its therapeutic efficacy [26].

The most traditional strategies to improve drug delivery at the tumour site through vascular or extracellular matrix remodelling or lay on pharmacological modulation of the tumour microenvironment But interestingly, increasing publications suggest that well-known physical approaches such as ionising radiations, hyperthermia, light irradiation or emerging ones like sonoporation, electroporation or exposure to cold atmospheric plasma (Figure 2) can be applied as a 
standalone strategy to 'prime the tumour' [27] or to end up with 'superenhanced permeability and

2 retention' (SUPR) effect [28]. Both results in an enhanced drug delivery and increased anticancer

3 efficacy which is practitioners' quest. Physical approaches offer particular advantages over

4 pharmacological or surgical approaches. They are less invasive than surgical intervention but their

5 major advantage is undoubtedly the targeting in time and space, which induces greater therapeutic

6 efficacy while drastically limiting the deleterious side effects that can be found with systemic

7 pharmacological treatments. This targeting in time and space presents two important consequences:

8 1) the impossibility of treating metastases because only tumours physically targeted by the treatment

9 are affected and 2) the existence of a therapeutic window which need to be precisely determined

10 during multimodal treatment with co-administration of nanotherapeutics. Other

11 advantages/disadvantages specific to the physical approaches described in this review are detailed in

12 Table 1.

13 This review will, thus, focus especially on these physical approaches (ionising radiations,

14 hyperthermia, light irradiation, sonoporation, electroporation or exposure to cold atmospheric plasma) and how they modulate vascular properties and degrade the extracellular matrix in order to improve the delivery of therapeutic molecules at the tumour tissue level. Wherever possible, examples demonstrating an improvement of drug delivery and efficacy through nanomedicine such as liposomes or polymeric nanoparticles will be developed. 

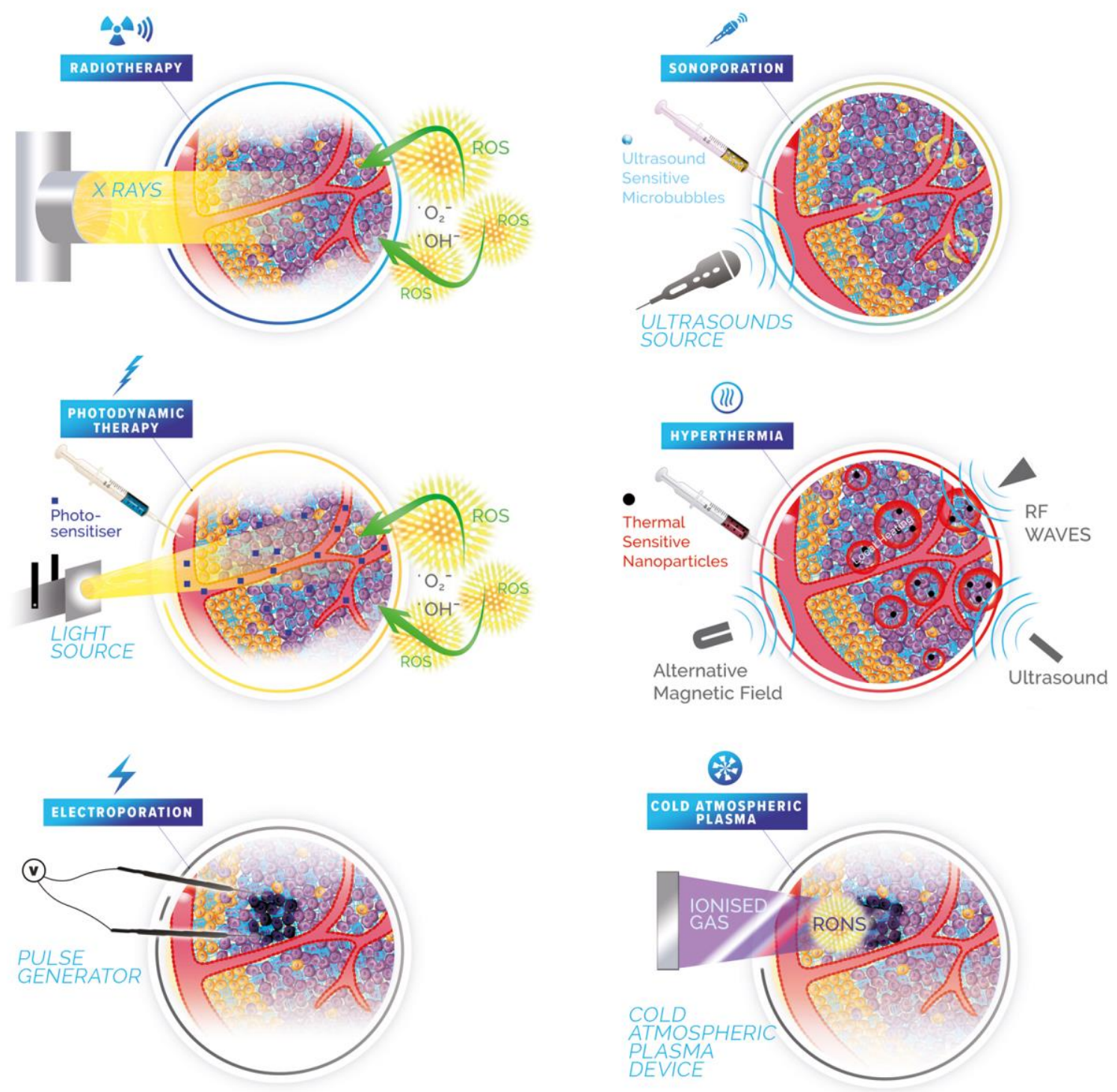

Figure 2: Remodelling the tumour microenvironment through physical pretreatments as emerging approaches in modern cancer therapy. Radiotherapy: water ionisation induced by X-ray leads to the production of reactive oxygen species (ROS) reknown genotoxicity and cytotoxicity on various cancerous cells types. Hyperthermia treatment: different types of HT source combined with responsive nanoparticles allow focusing the heat release into targeted areas while limiting adverse outcomes on the surrounding tissues. Photodynamic therapy: focused light irradiation excites a photosensitiser that promotes ROS production in the presence of oxygen, provoking cell death. Sonoporation: focused ultrasound associated causes microbubbles cavitation, a mechanical effect that improves ROS generation, membrane permeability and drug uptake. Electroporation: calibrated pulsed electric fields allow a local and transient permeabilisation of the cell membrane, leading to an enhanced drug uptake. Cold atmospheric plasma: interactions between low temperature plasma and liquids induce the production of short and long-lasting reactive oxygen/nitrogen species (RONS) responsible for its genotoxicity and cytotoxicity on various cancerous cells types. 


\begin{tabular}{|c|c|c|}
\hline & Advantages & Drawbacks \\
\hline All of them & $\begin{array}{l}\text { - spatial and timed control release } \\
\text { - decreased side effects compared to } \\
\text { systemic pharmacological treatments } \\
\text { - less invasive than surgical procedures } \\
\text { - Outpatient treatments }\end{array}$ & $\begin{array}{l}\text { - local treatment therefore does not reach } \\
\text { metastases } \\
\text { - therapeutic window needs to be precisely } \\
\text { determined during multimodal therapeutic } \\
\text { strategy }\end{array}$ \\
\hline Radiotherapy & $\begin{array}{l}\text { - highly focused, thus minimizing damage } \\
\text { or toxicity to adjacent tissues } \\
\text { - multiple sites treated in a single session } \\
\text { - patient satisfaction } \\
\text { - large clinical experience (over } 30 \text { years) }\end{array}$ & $\begin{array}{l}\text { - side effects: burns, fatigue, temporary } \\
\text { hair loss, sexual and fertility problems, } \\
\text { dermatological disorders, nausea and } \\
\text { vomiting, headache, trouble swallowing, } \\
\text { urinary issues... } \\
\text { - women are more sensitive to radiation } \\
\text { than men, but this is not considered in } \\
\text { international guidelines for radiation } \\
\text { dosages. }\end{array}$ \\
\hline Hyperthermia & $\begin{array}{l}\text { - highly focused, thus minimizing damage } \\
\text { or toxicity to adjacent tissues } \\
\text { - superficial and deep-seated tumors can } \\
\text { be treated }\end{array}$ & $\begin{array}{l}\text { - side effects: burns, blisters, discomfort, or } \\
\text { pain } \\
\text { - difficulty to keep the area to be treated } \\
\text { within a precise and homogeneous } \\
\text { temperature range for whole treatment } \\
\text { duration } \\
\text { - Lack of standardization depending on } \\
\text { kinds of energy used }\end{array}$ \\
\hline $\begin{array}{c}\text { Photodynamic } \\
\text { therapy }\end{array}$ & $\begin{array}{l}\text { - short treatment time } \\
\text { - possibility of repetitive treatments } \\
\text { - Selectivity (on the area receiving both the } \\
\text { photosensitizer + light irradiation will be } \\
\text { treated) } \\
\text { - aesthetic and functional healing } \\
\text { - cost-effective treatment } \\
\text { - great patient satisfaction }\end{array}$ & $\begin{array}{l}\text { - side effects: transient skin } \\
\text { photosensitivity, oedema, redness or } \\
\text { blisters formation } \\
\text { - correct oxygenation of the tissue is } \\
\text { essential } \\
\text { - accessibility for light delivery device can } \\
\text { be complicated depending on body site to } \\
\text { be treated } \\
\text { - limited light penetration } \\
\text { - Lack of standardization }\end{array}$ \\
\hline Sonoporation & $\begin{array}{l}\text { - highly focused, thus minimizing damage } \\
\text { or toxicity to adjacent tissues } \\
\text { - enhanced drug penetration effectively } \\
\text { - great patient satisfaction }\end{array}$ & $\begin{array}{l}\text { - side effects: irritation, burning } \\
\text { - Potentially time-consuming to administer } \\
\text { and expensive } \\
\text { - Lack of standardization }\end{array}$ \\
\hline Electroporation & $\begin{array}{l}\text { - highly focused (between electrodes), thus } \\
\text { minimizing damage or toxicity to adjacent } \\
\text { tissues } \\
\text { - enhanced drug penetration effectively } \\
\text { - possibility of repetitive treatments } \\
\text { - aesthetic and functional healing } \\
\text { - great patient satisfaction }\end{array}$ & $\begin{array}{l}\text { - side effects: muscle contraction, pain } \\
\text { - potentially time-consuming (electrodes } \\
\text { placement) } \\
\text { - expensive (disposable electrodes) }\end{array}$ \\
\hline $\begin{array}{c}\text { Cold } \\
\text { atmospheric } \\
\text { plasma }\end{array}$ & $\begin{array}{l}\text { - short treatment time } \\
\text { - cost-effective treatment }\end{array}$ & $\begin{array}{l}\text { - multiple parameters to control to ensure } \\
\text { desired plasma composition (loaded gas, } \\
\text { flow rate, voltage, exposure time...) } \\
\text { need for a constant distance to the } \\
\text { treatment site, limiting the treatment to } \\
\text { small areas } \\
\text { - depth of plasma penetration } \\
\text { - Lack of standardization }\end{array}$ \\
\hline
\end{tabular}




\section{Radiotherapy}

Nowadays, more than fifty percent of patients are treated routinely with radiotherapy (RT), associated or not with chemotherapy or other therapeutic modalities. The principle behind this technology developed in 1896 is based on a water ionisation process, leading to the production of reactive oxygen species (ROS). These tumoricidal doses of ROS provoke oxidative damages to DNA and to other cellular components in the tumour. As an additional positive consequence, RT modifies the tumour microenvironment by affecting endothelial cells and vasculature and remodelling extracellular matrix (ECM), but also counts negative impacts as it affects immune cells, and activates a number of pathways involved in tumour growth, dissemination and radioresistance $[29,30]$.

\subsection{Radiotherapy-Mediated Vascular Permeability Enhances Drug Delivery}

Precise radiation technology and their safer application has been made possible thanks to the progress made in the fields of radiation physics and computer technology. Mapping the tumour to deliver local ionising radiation with a radiation beam that matches the tumour shape has proven to be strongly impactful on the tumour vascularisation, the vessel permeability and the interstitial fluid pressure (IFP). The response of endothelial cells to radiation is accompanied by changes in transcriptional, translational processes and pro-angiogenic growth factor secretion. The observed effects on tumour blood vessels partly result from both a decrease in tumour volume and endothelial cells death by apoptosis. This causes vascular collapse and hypoxia at the tumour level as well as endothelial destabilisation. These effects can be observed both after RT treatment at low fractionated doses between 2-3 Gy [31,32] or for single high doses of radiation > $10 \mathrm{~Gy}$ [33]. It was observed that single radiation dose of $5 \mathrm{~Gy}$ or more induced, in a dose-dependent manner, a decrease of the vascular volume [34]. Moreover, in single-dose delivery protocols of moderate to high intensities, vascular damages resulted in an improvement of vascular permeability [34]. In order to demonstrate a radioinduced increase in vascular permeability, Schwickert et al. treated Fischer rats bearing R3230 
mammary adenocarcinoma xenografts with Gd-labelled albumin and a unique RT dose (15 Gy to 5 Gy) [35]. Thanks to MRI exploration, they demonstrated a dose-dependent increase in albumin accumulation in tumour tissue attributed to an increase of vessel permeability. However, contrary to high-dose radiation, a fractionated RT treatment led to an accumulation of macromolecules without visible vascular alterations [36]. Another additional positive impact was the observation of the increased vessel permeability and the increased secretion of cytokines as VEGF right after RT. As expected, this was followed by a decrease of VEGF blood concentration due to vessels regression [37]. Radiation-induced vessel permeability will partly depend on total dose radiation, fractionation schedules, the tumour type and the tumour vasculature level before RT.

Tumour reduction subsequently to radiation exposure leads to a resistance decrease to vascular flow and to a blood perfusion increase. High single-dose radiation between $10 \mathrm{~Gy}$ and $20 \mathrm{~Gy}$ increased blood flow by a factor of two and reduce the spatial variability of blood flow throughout the tumour volume [38]. The influence of IFP on the tumour microenvironment is closely related to tumour vascularisation because of hyperpermeability of blood vessels together with non-functional lymphatics. Although results may be inconsistent from one patient to another [39], RT may decrease IFP in the tumour as reported by several studies [40,41]. A high single-dose radiation of 15 Gy increased vascular permeability by $60 \%$ over three days and reduced IFP by $40 \%$ [41]. In response to radiationinduced cellular damages, survival mechanisms can be activated to compensate their cytotoxic effects. As described by Moeller et al., fractionated RT induced tumour cells death leading to an increase of the tumour perfusion and a burst of ROS due to waves of hypoxia and reoxygenation [42]. However, it was also reported that the hypoxia-inducible factor-1 (HIF-1), transcription factor regulating the responses to changes in tissue oxygenation, will be activated [42]. HIF-1 will mediate the release of endothelial pro-angiogenic factors such as VEGF to protect tumour endothelium against radio-induced vascular damage and to promote new vessels growth $[31,43,44]$. Activation of survival mechanisms to escape from the deleterious effects of RT as well as HIF-1 upregulation will contribute to the tumour radioresistance. 


\subsection{Radiotherapy Induces Extracellular Matrix Remodelling}

The dense extracellular matrix is an important component of the tumour tissue and is produced by stromal cells such as tumour associated fibroblasts, the main ECM producers. However normal fibroblasts are resistant to high doses of radiation up to $50 \mathrm{~Gy}$ [45]. Matrix metalloproteinase (MMPs) contribute to the turnover and modelling of the ECM. However, their activity may also be affected by RT as well as that of their inhibitors. Several in vitro studies have shown that radiotherapy increased MMPs activity and ECM proteolysis leading to an enhancement of tumour cells migration and angiogenesis. Many research groups have observed MMP-2 upregulation after different RT protocols and for various tumour types such as pancreatic [46], colon cancer (25 Gy in 5 fractions overs 5 days) $[47,48]$, lung cancer [49], breast cancer [50]. It has been suggested that MMP-2 may contribute to tumour cells dissemination $[47,51,52]$. In vitro studies performed on breast cancer cells [50] and rectal cancer cells $[47,51]$ support this hypothesis as they highlighted a higher concentration of MMP-2 after the application of RT which in turn led to an increase of the invasive properties of the tumour cells. Moreover, the inhibition of MMP-2 enhances tumour radiosensitivity in murine lung cancer $(2 \times$ 5 Gy) [49] while that of MMP-14 in murine breast cancer ( $3 \times 6 \mathrm{~Gy}$ ) [53] even enhanced the response to RT via an increased tumour perfusion. Some studies on rectal cancers showed an increase of MMP1, MMP-3, MMP-9 mRNA concentrations after RT $[47,54,55]$ and of PAI-I protein involved in radiation induced fibrosis $[54,48,56]$.

Lysyl oxidase (LOX), an enzyme involved in the crosslinks formation between collagens and elastin fibres, also plays a key role in cancer cell invasion through ECM remodelling [57,58]. LOX-activity inhibition has proven to be a relevant strategy to successfully prevent ECM stiffening and remodelling [59]. Unfortunately, RT promote, in a dose-dependent manner, an increase of the LOX secretion in several in vitro cancer cell lines and in vivo in a lung adenocarcinoma xenograft model [60]. Notably, knockdown of LOX in prostate cancer cells both in vitro and in vivo enhanced their radiosensitivity [61]. 
2 percent of cancer patients routinely, radio-resistance still occurs. However, radiobiologists are now considering seriously the importance of ECM remodelling and tumour microenvironment in general to achieve the expected outcomes of the treatments and overcome resistance and/or recurrence in different cancers [62].

\subsection{Radiotherapy Promotes Nanotherapeutics Delivery}

As explained here above, the tortuous nature of newborn blood vessels in the solid tumours together with the dense structure of the tumour ECM present significant impediments to blood-borne delivery of anti-cancer nanotherapeutics. The advantage of nanotherapeutics compared to traditional anti-cancer drugs rely on their small size which in practice favours the drug systemic pharmacokinetic. Indeed, due to their ability to escape from the mononuclear phagocyte system and to solubilise lipophilic drugs, researchers were expecting an increase of nanotherapeutic accumulation via the EPR effect. But clinical observations performed on three FDA approved nano-drugs clearly indicate modest benefits of nanotherapeutics compared to the standard drugs. Though their nano-size allows overcoming pharmacokinetic barriers, the complexity of the tumour microenvironment, the heterogeneous blood flow in the tumour, the dense collagen network of ECM and a high IFP in the tumour hamper their penetration on the battle field. Instead, therapeutics accumulate in the perivascular area. In this context, RT may achieve success in varying degrees by remodelling tumour microenvironment, and therefore, annihilate the posed obstacles. RT may also take advantage of nanomedicine design to overcome tumour resistance mechanisms and hypoxia [63]. Several studies on rat bearing Dunning AT1 prostate tumour have shown that single dose RT [64] or fractionated radiotherapy treatment (Figure 3) [65] before administration of HPMA copolymer-based nanovector loaded with doxorubicin (DOX) or gentamicin increased tumorous accumulation of nanotherapeutics and their therapeutic activity. 

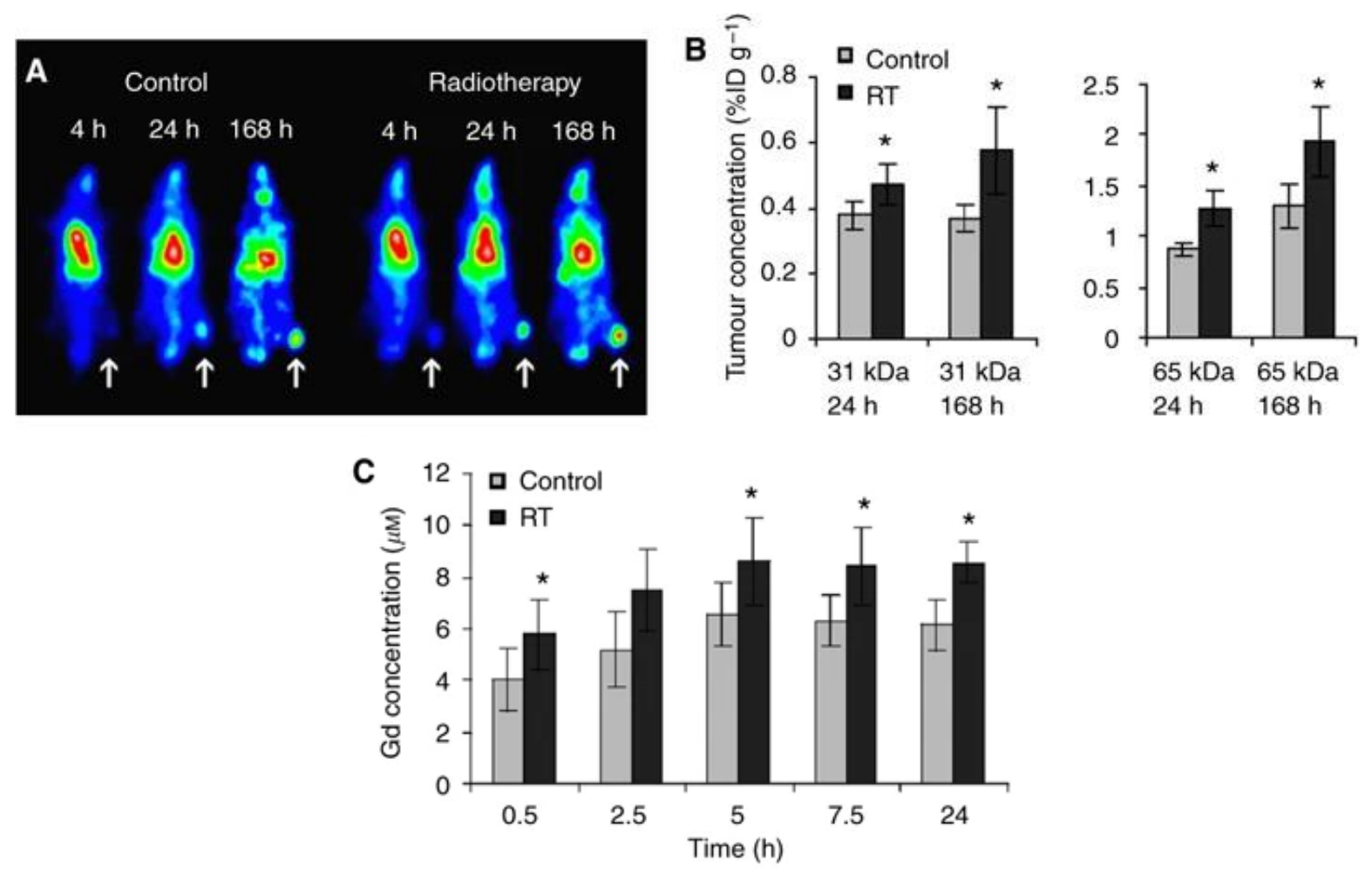

Figure 3: Radiotherapy (RT) improves tumor drug accumulation. (A) Scintigraphic analysis of the effect of $20 \mathrm{~Gy}$ of local tumour RT on the tumour accumulation of an iodine-131-labelled $31 \mathrm{kDa} \mathrm{N}-(2-$ hydroxypropyl)methacrylamide (HPMA) copolymers, demonstrating that RT beneficially affects tumour targeting. (B) Quantification of the effect of RT on the tumour concentrations of the $31 \mathrm{kDa}$ (left panel) and $65 \mathrm{kDa}$ (right panel) copolymer at 24 and $168 \mathrm{~h}$ post intravenous injection. (C) Quantification of the effect of RT on the tumour accumulation of the $25 \mathrm{kDa}$ gadolinium-labelled copolymer. Adapted from [65] under the Attribution-NonCommercial-ShareAlike 3.0 Unported (CC BYNC-SA 3.0).

Similar effects were observed with PEGylated liposomal doxorubicin (Caelyx) when athymic mice bearing human osteosarcoma xenografts were previously exposed to a single dose of $8 \mathrm{~Gy}$ or fractionated radiotherapy ( $3 \times 3.6$ Gy) [66]. Giustini et al. showed an increased accumulation of iron oxide nanoparticles in the tumour after a single 15 Gy irradiation in a syngeneic mouse breast cancer model [41]. For each example, authors demonstrated that this increase in nanotherapeutics accumulation was correlated to a decrease of the IFP and an enhancement of vascular permeability. Recently, Stapleton et al. clearly demonstrated how IFP may modulate accumulation of nanotherapeutics in a tumour previously exposed to RT [67]. In a preclinical tumour model with high IFP, the MDA-MB-231 metastatic breast adenocarcinoma model, a single dose of radiation (15 Gy) promoted a decrease of the IFP by $50 \% 24 \mathrm{~h}$ after RT. Tumour microenvironment modifications induced by RT disinhibit interstitial transport of larger carriers such as liposomes, mostly in the central area, 
allowing an improved cellular uptake of the liposomal doxorubicin (Doxil) and an enhancement of the doxorubicin therapeutic efficacy. This study pointed out that RT causes spatiotemporal fluctuations in fluid transport, mediating the decrease of the IFP and subsequent nanoparticles accumulation in tumours.

Interestingly, RT was also proved to improve nanotherapeutics (PLGA-PEG nanoparticles, Doxil or Onivyde) accumulation within tumor indirectly through the recruitment of tumor-associated macrophages (TAM) acting as nanoparticles carriers [68]. Thus, TAM recruited on site by RT elicited dynamic bursts of extravasation, and subsequently enhance drug uptake in neighboring tumor cells.

All these results support the clinical investigations combining RT pretreatment with nanotherapeutics administration for the treatment of cancers. Phase I/II clinical trials testing liposomal doxorubicin, cisplatin or oxaplatin formulation in combination with radiotherapy have shown better patient's response and increased patients' survival while they were affected by lung cancer, bladder cancer, breast cancer, and head and neck cancers associated $[69,70]$. In a phase I study using poly (Lglutamic acid)-paclitaxel anti-cancer drug combined with 6 weeks RT exposure at 50.4 Gy, a complete clinical response for four out of 12 patients (33\%) affected with loco-regional disease was observed [71]. Meaning that no tumour was visible as assessed during follow-up endoscopy and post-treatment biopsy. These promising clinical results pave the way for the development of new multimodal therapies combining both pretreatment of the tumour microenvironment and the delivery of nano-sized chemotherapeutic agents.

Structure and nature of the tumour microenvironment will influence the tumour response to RT. The many examples described above and in the literature demonstrate the great potential of RT to impact tumour vasculature, decrease IFP and degrade collagen proteins from the ECM. Therefore, one can understand that patient's recovery as well as RT resistance and tumour recurrence will depend on this interplay between tumour response to RT and ability of RT to denature the tumour microenvironment. In case that prove to be effective, RT may be a powerful tool for nanotherapeutics delivery and their intratumoral accumulation. While the multimodal strategy combining RT and nano- 
drugs appears promising clinically, the next challenging steps would be to evaluate the influence of the sequence and timing of nanotherapeutic and RT administration, the specific fractionation schedule, the total radiation dose and dose per fraction on the tumour response.

\section{Hyperthermia}

The principle of hyperthermia (HT) treatment grounds on the regio-local elevation of temperature in the tumour tissue to be treated to induce either reversible or irreversible damages. Currently, accepted temperature to be reached by this technique is ranging from 39 to $43^{\circ} \mathrm{C}$ [72]. Today this topic is well documented and, for thorough description, we invite the reader to go through the following reviews and references $[8,73-75]$. Historically, the local increase of temperature in the tumour was assessed by physicians as being the best way to cure cancer. However, they quickly realised that temperatures higher than $43^{\circ} \mathrm{C}$ led to irreversible side effects such as haemorrhage and stasis. That is the reason why most studies currently refer to what is called mild hyperthermia, aiming at a moderate elevation of temperature $\left(39-43^{\circ} \mathrm{C}\right)$. In a bid to unravel the biological mechanisms behind the thermal cytotoxicity, intense investigations were carried out from the last mid-20th century. Better understanding of molecular interaction pathways on ECM proteins, DNA damages repair as well as its role in immunomodulation allowed to refine HT protocols. In addition, thanks to the significant development of technologies and software enabling online thermometry and treatment planning, safer application of HT was made possible to be used as a potent cure technique. To control locally the temperature, different physical means have been developed e.g. high-intensity focused ultrasounds (HIFU), microwaves, light irradiation of photo-responsive nanoparticles (mainly composed of gold), oscillating magnetic field on metal nanoparticles and various radiofrequencies. The use of external electromagnetic fields presents the strong advantage to have a better control on heating localisation. It implies that sensitive nanoparticles that can be activated at a distance are distributed onto the site to be treated. This is the case for plasmonic photothermal therapy and magnetic hyperthermia. In such cases, the sensitive nanoparticles are used as physical tools that trigger the stimulus while the EPR 
effect promote their accumulation in the tumour surroundings. In a complementary manner, hyperthermia has been intensely used jointly with thermosensitive liposomes carrying regular anticancer drugs. In this case, the liposomes [73] do not generate hyperthermia but they react to it and subsequently deliver the drug. Such tandem application is so effective that it is currently evaluated in several clinical trials [8], some of them at phase III. In the following paragraphs, hyperthermia effects emphasis will be done first on the vascular system and secondly on ECM remodelling.

\subsection{Vascular Effects of Hyperthermia are Numerous}

Loco-regional vascularity change is the direct consequence of HT applications. Regardless of which cells are being treated, gradual rise of temperature above $39^{\circ} \mathrm{C}$ induced increase blood flow. However, if an increase of blood flow by a factor of 15 , at least, is measured for healthy tissue, this effect is reduced to a factor of 2 for tumour tissue because temperature distribution inhomogeneity within the tumour and the tumour type [76]. Nevertheless, the very first important effect observed when HT is applied near a tumour is the increase of extravasation of different molecules or nanoparticles, linked to an improved permeability of the endothelial wall [77-79]. For instance, Ferrari et al. described the transport of Evans blue and different dextrans in pancreatic subcutaneous tumours and observed an increased perfusion with a mild hyperthermia persisting at least 5 hours but coming back to normal 24 hours later. A similar transient effect was observed by Koning on melanoma and sarcoma [80]. In an elegant work, Hu et al. developed nanoplatforms including nanoparticles based on carbon nanotubes, chitosan and nanogels composed of the thermoresponsive poly ( $\mathrm{N}$-isopropyl acrylamide) (PNIPAM). PNIPAM exhibits a tunable water solubility transition with temperature called Lower Critical Solubility temperature (LCST) and around $32^{\circ} \mathrm{C}$. Below this threshold, the polymer remains soluble while it precipitates above this value. When employed to design nanovector, PNIPAM enables to tunable nanovector size with temperature. In this case, the nanoplatform transported doxorubicin (DOX). Upon local heating, the nanoplatform shrank due to the LCST and collapsed nanostructure benefited in an even stronger way of the increased permeability of the blood vessel. Interestingly, the system was also 
$\mathrm{pH}$-sensitive and typical acidic environment in the tumour led to its protonation, which has for consequence to provoke their adhesion onto cancer cells [81].

However, and as underlined here-above, moderate increase of blood flow in tumours was observed, as described more than 20 years ago by Baronzio et al. [82], and recently confirmed by Dynamic Contrast Enhanced MRI characterising the delivery of doxorubicin from thermosensitive liposomes to mammary carcinoma. No increase of blood flow rate was observed even though an increased drug delivery could be seen [83]. Recent investigations highlighted that longer exposure to temperature above $42.5^{\circ} \mathrm{C}$ could result in impairment of blood flow since fragile and chaotic neoangiogenic tumour microcirculation is severely damaged $[76,84]$. In some extent, a complete shutdown of the vasculature can be observed, proving the thermal toxicity of HT on tumour cells.

Upon increasing temperature over $44^{\circ} \mathrm{C}$, various studies repetitively described predictable deformation of the vessels, stiffening of red and white blood cells, leading to their sticking to the wall, coagulation [85] and haemorrhage $[83,86,87]$.

Regarding vascular effects of hyperthermia, a point is still under debate. Different studies have described the development of a thermoresistance process, upon which hyperthermia effects are lost in a second treatment $[88,8]$. However, Raucher's group consistently used four cycles of hyperthermia on pancreatic tumours and showed an increase of penetration of elastin-like peptides [89]. Gazeau et al. also described the treatment of epidermal carcinoma thanks to heat-generating iron oxide nanocubes following a protocol of 3 cycles of exposure to oscillating magnetic field [90].

Considering heterogeneity of temperature distribution within the tumour, this point is almost never examined in the recent literature. Realistic therapeutic strategies should thus be elaborated for HT translational clinical applications, taking this point into account.

\subsection{Hyperthermia Based on Nanoparticles Is an Efficient Way to Remodel ECM}

As discussed in the introduction of this review, ECM is an essential element to consider for the development of oncologic approaches as tumour stiffness and ECM density impair anti-cancer drug 
delivery seriously. Even if the current therapies still underestimate this target in their therapeutic armamentarium development, its role in limiting drug delivery to tumours has been recognised for a long time and strategies to overcome the resulting impairments have been proposed, based on either chemical or physical approaches $[87,91]$. Penetration of ECM is an even more challenging barrier from the view point of nanotherapeutics as their convection-based transport is restricted by the dense protein entanglement of the ECM and tumour stiffening. The use of hyperthermia to alleviate this problem is still not magic, as demonstrated by Koning in 2017 who compared hyperthermia treatment on two different breast cancers [92]. The therapeutic response of thermosensitive liposomes loaded with DOX was better for the cell type which presented a higher blood vessel density and a poorly organized ECM.

Increasing information has been presented on existing links between matrix stiffness and cancer prognosis [86]. Indeed, the aberrant production of ECM leading to tumour stiffening has been recognised as a predictive marker of tumour malignancy. In an on-demand tunable ECM model composed of collagen gel, several studies have examined the influence of temperature on such a simple system. For instance, Chan et al. used a microfluidic set up to evaluate the penetration of $50 \mathrm{~nm}$ and $120 \mathrm{~nm}$ gold nanoparticles inside a collagen gel incorporating gold nanorods [93]. They observed the denaturation of type I collagen fibrils at $45-55^{\circ} \mathrm{C}$ and an increased diffusivity of both types of nanoparticles. Collagen is known to exhibit several phase transitions upon heating, a minor transition existing at ca. $31-37^{\circ} \mathrm{C}$ and a stronger and irreversible one in the $37-55^{\circ} \mathrm{C}$ range [90]. Mild hyperthermia thus inevitably leads to denaturation of collagen gels and, in turn, allow ECM remodelling. This has repeatedly been described in the literature when people precisely examine this point. For instance, Hilger et al. studied the evolution of collagen fibres in mixed spheroids of Panc-1 pancreatic cancer cells and WI-38 fibroblasts [94]. They demonstrated a marked evolution of collagen fibres above $39{ }^{\circ} \mathrm{C}$ and a decrease of collagen content inside spheroids. A further increase of temperature to $40{ }^{\circ} \mathrm{C}$ led to a decrease of the spheroid size and even to their disintegration at $55^{\circ} \mathrm{C}$. Similarly, another study described that hyperthermia performed on tissue-engineered human dermal 
substitutes loaded with magnetic iron oxide nanochains provoked cell death and collagen melting [95].

The nanochains were either found in endo-lysosomes or in extracellular vesicles.

In order to demonstrate the added value of hyperthermia for doxorubicin (DOX) delivery, Ta et al. compared the therapeutic efficiency of either free DOX, DOX encapsulated in thermosensitive liposomes and DOX in thermosensitive liposomes modified by the already mentioned thermosensitive polymer PNIPAM [96]. Interestingly, the best results on adenocarcinoma were obtained for the thermosensitive system modified by the PNIPAM polymer and these were associated with a more important remodelling of ECM.

In an original work, Guttierez et al. compared two in vitro models of magnetic hyperthermia [97]. The first one contained iron oxide nanoparticles only inside phagocyte cells (macrophages or monocytes) whereas in the second one, nanoparticles were present both inside and outside these cells located in the collagen matrix. When nanoparticles were present both inside and outside the cells, an ECM disruption was observed which enabled a faster homogeneous distribution and a deeper penetration of the nanoparticles in the 3D culture. Furthermore, both models exhibited different cell death processes, necrosis being mostly observed when nanoparticles were located only inside the cells whereas apoptosis was predominant for the other model.

Notably, Gazeau et al. showed that upon illumination a transient stiffness increase was observed in vivo during photothermal therapy on epidermoid mouse carcinoma. Right after the treatment, a neat decrease of the stiff areas as well as a volume reduction of the tumours, either under mild hyperthermia or photoablation conditions were detected [86].

Recently, degrading ECM not only with heat-generating nanotherapeutics but also with enzymes that can cleave collagen has been proposed as a potent synergetic approach for ECM digestion. Pu proposed to encapsulate bromelain in photoresponsive particles [98]. Bromelain is a protease enzyme which becomes active around $45^{\circ} \mathrm{C}$, therefore, only upon heating. This has the enormous asset to avoid possible danger of destroying ECM in healthy tissues. Another similar study was presented by Städler in 2019, in which, microswimmers with adsorbed collagenase had the property to move 
towards low calcium concentration areas [99]. That assembly enabled them to penetrate more deeply towards the center of tumour spheroids which is an important breakthrough considering the intrinsic difficulty to penetrate in the core of a solid tumour.

High-intensity focused ultrasounds (HIFU) is a novel therapeutic strategy based on acoustic energy for the thermal ablation of deep-seated tumours. While still under development, proof of concept studies demonstrated that this HIFU-based moderate HT allows higher temperature uniformity within the tumour [100]. In mice skeletal muscle, pulsed-HIFU were found to enhance the delivery of systemically administered fluorescently labelled nanospheres (100 nm or $200 \mathrm{~nm}$ ). These observations correlated with transient (reversed within $72 \mathrm{~h}$ ) structural histologic alterations such as enlarged gaps between muscle fibre bundles and a disruption of the connective tissue [101]. Lee et al. demonstrated that using pulsed-HIFU in mice bearing human tumour xenografts rich in ECM resulted in an increased blood flow, an ECM remodelling with decreased collagen contents and a 2.5 times higher enhanced penetration of glycol chitosan nanoparticles than in untreated tumours [102]. According to the authors, the ECM disruption may be attributed to the acoustic cavitation and radiation forces generated during exposure to focused ultrasounds. This demonstrates the relevance of pulsed-HIFU to enhance tumour targeting of nanomedicines via ECM remodelling. This subject will be dealt with in more details in the sonoporation section.

2.3 Hyperthermia is Increasingly Used Jointly with Another Therapeutic Method in 2-step

\section{Protocols}

Hyperthermia is a multi-action therapeutic modality whose positive effects are the vascular permeability increase, moderate blood flow augmentation, remodelling of ECM, partly through disruption of collagen fibrils network. However, the necessity of limiting the temperature below $43^{\circ} \mathrm{C}$ to avoid severe irreversible damages as haemorrhage and stasis has impeded the development of hyperthermia as a standalone technique. Since the all-above-mentioned benefits prime the tumour 
environment, novel approach in contemporary oncology practice is to combine HT with other tumour care as radiotherapy or chemotherapy (CT) for a subsequent and improved tumour eradication [88]. Examining the recent literature proves that this is the current path followed. The strategy aims to destabilise ECM in a first step followed by adequate treatment to promote cancer cell death. In such protocols, hyperthermia can either be used as the first or the second step. In a study reported by Pang et al., the anti-cancer drug cyclopamine was administered to mice bearing pancreatic ductal adenocarcinoma for 3 weeks before injecting gold nanorods [103]. Cyclopamine led to ECM destructuration, improving the outcomes of the subsequent photothermal therapy.

In another outstanding research example, Li et al. described the development of two bioinspired lipoproteins systems (bLP) (Figure 4). The first one (D-bLP) bore a photoresponsive group enabling photothermal treatment remodelling ECM and modulating stromal cells. The second lipoprotein system was based on encapsulated mertansine (M-bLP), an anti-cancer drug. Upon consecutive treatment, a 27-fold increase of accessibility of mertansine to the cancer cells was observed. Interestingly, migration of cancer cells was strongly limited, as well as cancer stem cells, limiting therefore self-renewal capacity. Even more importantly, this protocol led to a $97.4 \%$ inhibition of lung metastasis [104].

From a general standpoint, hyperthermia has evolved in the years from a possible standalone technique to a powerful method when used jointly with another therapy. These approaches together with realistic clinic amendments aim at restricting temperature increase to reach a range tolerable for the patients without side effects. Even if we are still far from the 'bench-to-bedside' application, the multifaceted actions of $\mathrm{HT}$, including increased vascular permeability, ECM remodelling and interaction with a wide range of anticancer drugs, is now recognised as a powerful tool with huge potential to improve cancer patients cure without significantly late or acute tissue morbidity. 
a

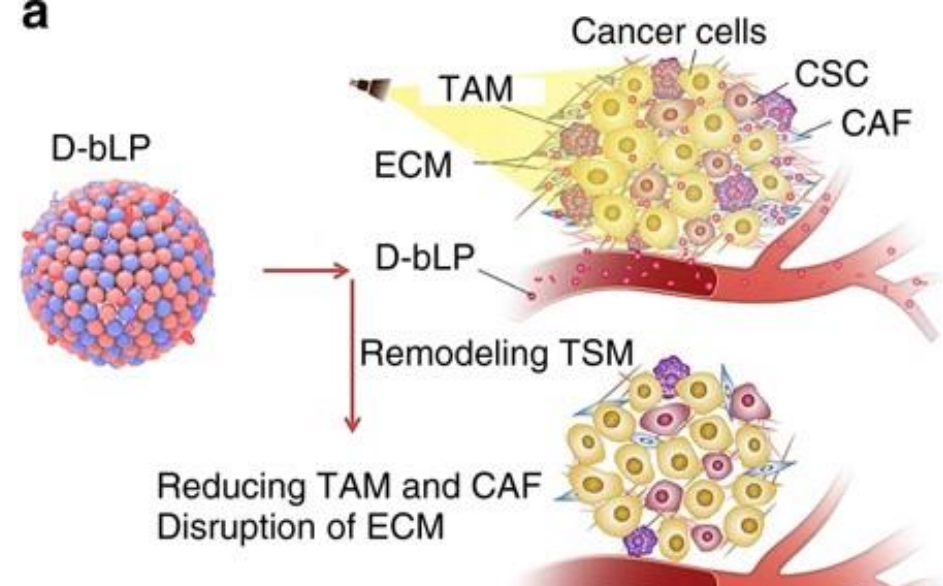

b

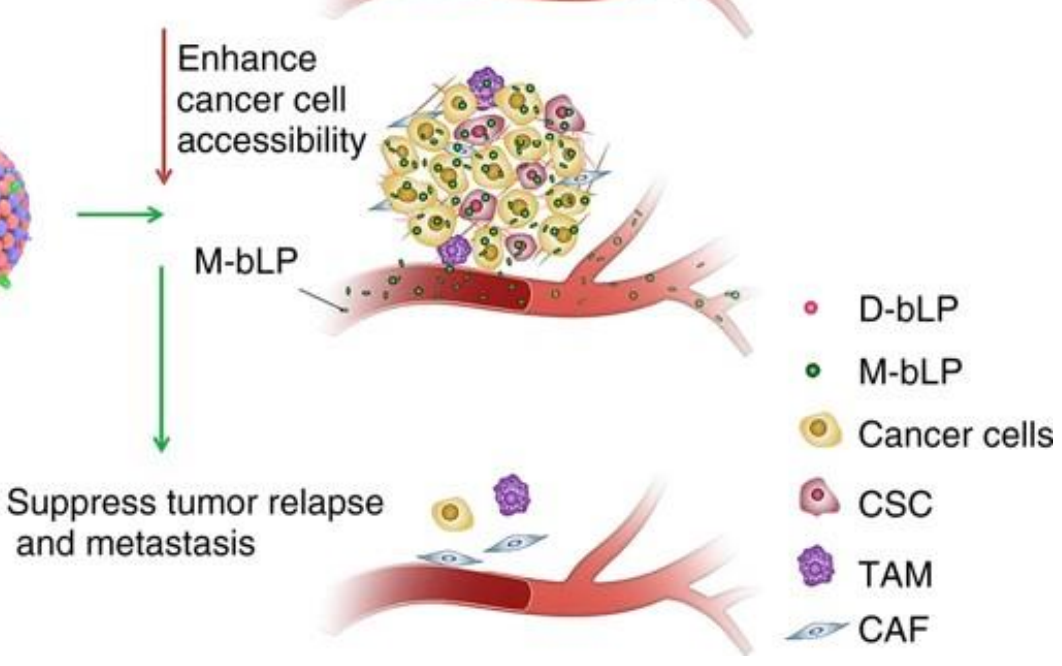

Figure 4: Schematic illustration of D-bLP-mediated photothermal remodelling of tumour stroma to enhance second M-bLP accessibility to cancer cells. A. D-bLP-mediated photothermal effects cause drastic elimination of stromal cells (e.g., CAF and TAM) and ECM components (e.g. collagen I, fibronectin). b D-bLP-mediated tumor stroma microenvironment remodelling enhances the accumulation and penetration of second M-bLP in tumours, promotes their extravasation from tumour vasculature and accessibility to cancer cells, thus resulting in efficient suppression of tumour relapse and metastasis. bLP bio-inspired lipoprotein; M mertansine; CSC cancer stem cells. From [104] under the Creative Commons Attribution License (CC BY 4.0).

\section{Photodynamic therapy (PDT)}

In this section we will focus on photodynamic therapy instead of light irradiation broadly speaking. Indeed, light irradiation with lasers and light-emitting diode (LED) sources are classically used in perspective of skin rejuvenation, aiming at reducing wrinkles by stimulating a deposition of substantial amounts of collagens, which defeats the goal of improving nanotherapeutics delivery in the frame of cancer therapy. Photodynamic therapy (PDT) is a nonthermal therapeutic modality based on nontoxic 
photoactive photosensitiser molecules that spatiotemporally produce reactive molecular species such as reactive oxygen species (ROS) at the site of light irradiation [105]. The tumoricidal dose of ROS is only delivered after activation or 'turned on' when a certain kind of light irradiates the site of treatment and provoke tumour cell death by apoptosis or necrosis. To be effective, PDT requires three essential components, the photosensitiser, the corresponding light source and oxygen. PDT is now commonly used in the clinic stage for dermatological, ophthalmologic and oncologic applications. PDT using short time interval between photosensitiser injection and light irradiation, called short drug-to-light interval, mainly destroys tumour vasculature by confining photosensitiser localisation within blood vessels, whereas long interval between photosensitiser injection and light irradiation can induce more damages to tumour cells, because the photosensitiser has then been distributed into the extravascular compartment. Since its efficiency is strongly related to areas light can reach, PDT is particularly adapted to superficial cancer, located right under the skin or in the linings of organs that can be reached with light. Despite this Achilles' heel, this outpatient procedure can be applied as a pretreatment in combination with other anti-cancer therapies to increase outcomes for patients. PDT is becoming increasingly successful as it is minimally or not invasive, fast and with light side effects, with the possibility of focusing the light beam on the area to be treated with a superior level of precision. Thus, next sections will describe how PDT can affect vascular compartment and ECM remodelling, in the perspective to move drug delivery at the tumour site up a notch.

\subsection{PDT-Mediated Vascular Permeabilisation Enhances Drug Delivery}

Beyond its tumoricidal activity, this light-activated therapy leads to significant vascular damages since the endothelial cells lining blood vessels are in the front line against the photosensitisers, most commonly injected intravenously.

PDT-induced damages to the endothelium trigger a cascade of physiological events including platelet aggregation, the release of vasoactive molecules, localised inflammation, adhesion of 
leukocytes, increased vascular permeability (which has significant potential to increase the diffusion of drug molecules to the tumour) and constriction of vessels [106]. The mechanisms leading to endothelial damages are photosensitiser-dependent [107]. Using in vivo photofrin-based PDT on rat cremaster muscle, Fingar et al. demonstrated a dose-related constriction of arterioles, observed within the first minutes of $630 \mathrm{~nm}$ illumination, associated with an increase in venule permeability to albumin occurring shortly after the start of light treatment and was progressive with time [108]. Fluid leakage from vessels is also observed after PDT as demonstrated by oedema formation and the increase in tumour interstitial pressure [109].

In vitro transmission electron microscopy experiments in light-irradiated bovine endothelial cells treated with $25 \mathrm{mmol} . \mathrm{L}^{-1}$ haematoporphyrin revealed endosomal and lysosomal membrane disruption, endoplasmic reticulum swelling and nuclear membrane swelling associated with chromatin degradation [110]. Leunig et al. demonstrated that as early as 15 minutes after being subjected in vitro to PDT with photofrin, HUVECs showed a $140 \%$ increase in volume [111]. One hour after PDT, blebs appeared on their surface, accompanied by a $20 \%$ decrease in the number of viable cells. Sporn et al. demonstrated on HUVECs that photofrin $(1 \mu \mathrm{g} / \mathrm{mL})$ induced a light dose-dependent depolymerisation of the microtubules as early as 15 minutes after light irradiation while the actin microfilaments were not affected [112]. It is conceivable that the increase in vascular permeability observed in vivo after PDT correlates with cytoskeletal remodelling and endothelial cell swelling.

As PDT targets the tumour vasculature, inducing among other effects vascular permeabilization, it has been proposed to use PDT to enhance delivery of nanoscale therapeutics. For instance, PDT based on chlorin-based 2-[1-hexyloxyethyl-]-2-devinyl pyropheophorbide-a (HPPH) was used as a method to improve the delivery and efficacy of oncolytic vaccinia virus to primary and metastatic tumours in mice [113]. PDT-induced vascular disruption resulted in an over 10-fold increase intratumoral viral titres compared with the untreated tumours. However, depending on the light dose, PDT was more or less effective, underlining the need of optimisation when using PDT to increase intra- 
1 2 permeabilize vessels in order to facilitate the delivery of macromolecular agents as observed with

tumoral accumulation of therapeutics. A study by Snyder et al. confirmed the ability of PDT to fluorescent microspheres with diameters ranging from 0.1 to $2 \mu \mathrm{m}$ and liposomal formulation of doxorubicin (Doxil) $(0.1 \mu \mathrm{m})$ [114]. Using a murine colon cancer model, they showed that low fluence rate PDT prior to Doxil administration significantly increased DOX content in tumours, as well as ensured up to $80 \%$ long-term tumour control without concomitant enhancement of systemic or local toxicity. In their study they also underlined the importance of time interval between PDT treatment and Doxil administration. Thus, the maximum Doxil uptakes were found when the injection occurs immediately after the application of the PDT. Tumour vessel modulation by low-dose photodynamic therapy was shown to enhance the extravasation of macromolecular compounds such as liposomal doxorubicin (Liporubicin) into tumours [115]. It was also demonstrated on a lung sarcoma metastasis model that low-dose photodynamic therapy significantly decreased tumour interstitial fluid pressure without affecting tumour blood flow, thus promoting liposomal doxorubicin (Liporubicin) distribution into tumour (Figure 5) [116].
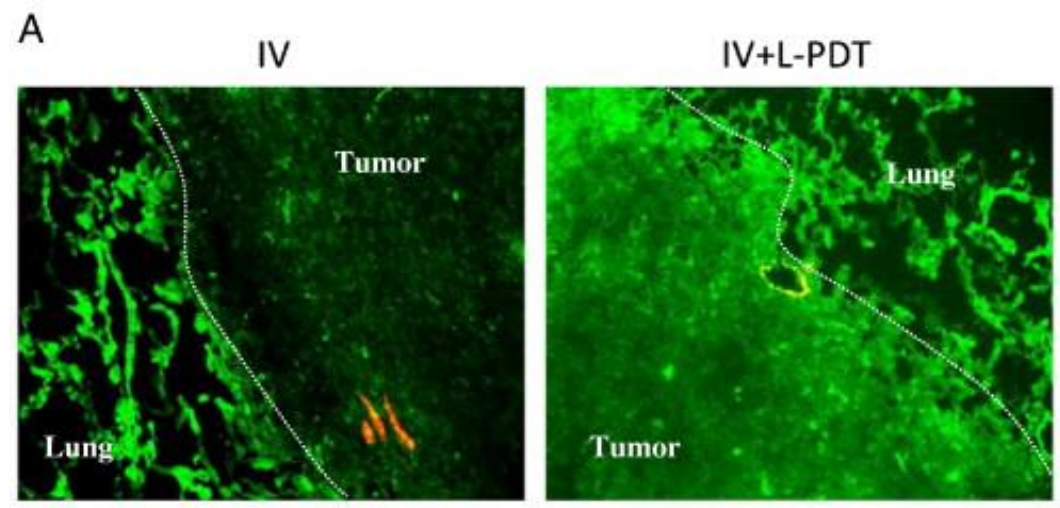

\section{B}

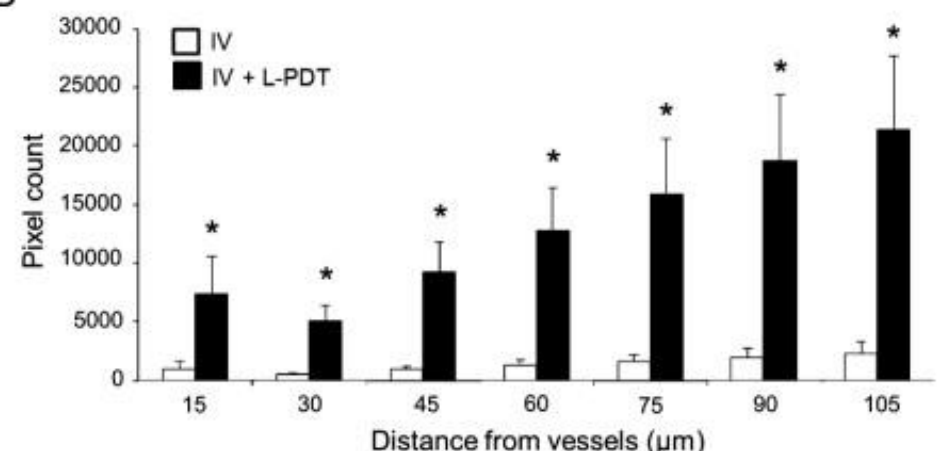


Figure 5: (A) Liporubicin fluorescence reconstruction images in tumors after intravenous (IV) administration of $400 \mu \mathrm{g}$ of Liporubicin with and without low dose photodynamic therapy (L-PDT) pretreatment (original magnification, $\times 40$ ). The green pseudocolor represents Liporubicin signaling, and the red pseudocolor represents tumor blood vessels. L-PDT pretreatment enhanced the distribution of Liporubicin in the tumor interstitium but not in lung tissues. (B) Liporubicin signaling quantification in the tumor at increasing distances $(\mu \mathrm{m})$ from the tumor vessels with and without LPDT pretreatment is shown. From [116], Copyright (2020), with permission from Elsevier.

Similarly, photoimmunotherapy induced in vivo a large increase in tumour vascular permeability, allowing a 5-fold increase in the accumulation of a liposomal daunorubicin (DaunoXome) and resulted in more effective therapy than either photoimmunotherapy or liposomal daunorubicin alone [117].

In conclusion, PDT is commonly used in clinics for its direct and well-known cytotoxic effects on tumour cells but it is also evolving as a new modality to improve the extravasation of nanomedicine through the permeabilized vascular compartment. As with radiotherapy, excessive PDT-induced damages to the vessels risk to shut down blood flow, negatively affecting drug delivery. Even if optimisations in terms of concentrations and delay between PDT treatment and nanoparticles administration have to be carried out, the PDT-mediated vascular permeabilization has clearly been demonstrated to enhance the accumulation of nanomedicine into tumours for enhanced efficacy. These evidences pave the way for an emerging combined therapeutic approach named nanoparticlemediated chemophototherapy [118].

\subsection{PDT Induces Extracellular Matrix Remodelling}

The dense and abnormal stiffness of the tumour ECM are responsible for blood vessels squeezing, reducing oxygen supply and drug delivery to solid tumours and oxygen diffusion hindering further limiting the therapeutic efficacy of PDT. Nevertheless, dermatological studies indicated improved wound healing and scar remodelling when repetitively treating skin wounds and scars using low dose PDT. Lv et al. analysed the effect of multi-session (once a week for a total of 12 times) of topical 5aminolevulinic acid (ALA)-based PDT on modulation of collagen components and structure in a hairless mouse model by second-harmonic generation [119]. Their results indicated that 12 sessions of PDT led 
to skin rejuvenation by improving dermal collagen density and its arrangement in skin. Mills et al. reported on 27 healthy donors that methyl aminolaevulinate-based PDT following excisional wounding (three treatments over 5 days) resulted in increased MMP1, MMP9, and TGF- $\beta 3$ production after 3 weeks, as well as a greater, more ordered deposition of collagen I, collagen III and elastin 9 months after wounding [120]. In the same line, it was demonstrated in vitro on human keratinocytes $\mathrm{HaCaT}$ cells that chlorin e6-based PDT promoted collagen production and suppresses MMP-1,-2,-9 expression [121].

Since hypericin exhibits a stronger affinity to collagen than chlorin e6 [122], it was proposed as a more effective photosensitiser in collagen-rich tissues, such as skin or tumours. Using fluorescence spectroscopy and multiphoton microscopy, Hovhannisyan et al. demonstrated in vitro in collagen gels [123] and in native tissues such as chicken tendons and skin [124] that hypericin - based PDT induced photosensitised irreversible destruction of collagen-based tissues.

Ferrario et al. demonstrated on extracts from photofrin-based PDT-treated tumours a strong expression of MMPs and extracellular MMP inducer (EMMPRIN) along with a concomitant decrease in expression of tissue inhibitor of metalloproteinase-1 (TIMP-1), suggesting that MMP-9 released by endothelial and inflammatory cells plays an active role in regulating the tumour microenvironment [125]. The sublethal doses of ALA and $580-740 \mathrm{~nm}$ light irradiation on human dermal fibroblasts was shown to in vitro induce matrix metalloproteinase 1 and matrix metalloproteinase 3 expression in a singlet oxygen-dependent way while reducing collagen type I mRNA expression [126]. In vitro treatment of primary human vocal fold fibroblasts with sublethal $(200 \mu \mathrm{M})$ ALA-based PDT was shown to significantly alter the expression of genes related to ECM remodelling [127]. Thus, the expressions of TGF-b1, COL1A2, COL3A1, fibronectin and elastin were reduced, while the expression of MMP1 was increased. The migratory capacities of fibroblasts were reduced, as well as the percentage of contractile aSMA-positive myofibroblasts in the population. Interestingly, a meta-analysis on early oral and laryngeal cancers clinically treated with photodynamic therapy confirmed that PDT results in no glottic 
scarring as compared to conventional lasers or surgical excision or vocal cord stripping [128], indicating that ECM remodelling correctly occurred after PDT.

In conclusion, the ability of PDT to efficiently remodel ECM through direct photosensitisation of collagens by the photosensitiser or the modulation of both collagens production by cells and MMPs activity may confer benefits to the drug delivery to the tumour. However, the proper therapeutic window (in terms of photosensitiser dose and timing of administration) must be identified since sublethal doses seem likely to be used. Furthermore, the necessary presence of a photosensitiser in this strategy is not without risk for the patient, particularly in terms of side effects such as skin sensitivity to bright light and sunlight, redness or even blisters formation. Besides this collateral effects, combination of PDT with other therapies offers many benefits compared to PDT monotherapy itself to eradicate tumour cells. Enhancement of drug delivery of the targeted site can also bring further enhancement of the PDT itself since physiological barriers arising from a tumour microenvironment can be raised during combined treatment to supply enough oxygen and photosensitiser on the right place to produce high enough dose of tumoricidal ROS. Recently, Ihsanullah et al. combined chemotherapy with PDT to design acidic activatable and externally induced hypoxia carriers to effectively eradicate tumour cells [129].

\section{Emerging physical techniques in cancer research promoting drug delivery}

\subsection{Sonoporation}

Contrary to high-intensity focused ultrasound (HIFU) therapy that uses focused ultrasound waves to thermally ablate a portion of tissue as described in the hyperthermia section, sonoporation induces the transient and reversible cell membrane permeabilization produced by local ultrasound exposure associated or not with microbubbles [130]. This phenomenon is used to facilitate the transport of drugs, nucleic acids and proteins into the cytoplasm [131]. Microbubbles, popular as ultrasound contrast agents, may also be employed as therapeutic carriers for localised, targeted drug or gene 
delivery by sonoporation $[132,133]$. These microbubbles are gas-filled structures stabilised or not by a shell composed of lipids, proteins or polymers. While for imaging applications low intensity of ultrasound is required in order to preserve microbubbles integrity, when applied for drug delivery purposes the ultrasounds are used to locally disrupt microbubbles according to the cavitation process, thus releasing their content when triggered [121]. Several studies have reported vascular and extracellular matrix modulation after sonoporation applications.

Cavitation of microbubbles following exposure to ultrasounds was shown to alter vascular integrity, allowing the release of circulating molecules. This observation underpinned the extension of sonoporation to be applicable as an anti-cancer treatment. The mechanism is not precisely known but it is hypothesised that the oscillations of cavitating microbubbles generate mechanical forces on the vessel wall and a concomitant permeability and molecule transport improvements [135]. When applied in vivo, microbubbles exposed to low-frequency ultrasound have been shown to cause rupture of microvessels accompanied with extravasation of red blood cells [136]. Stable cavitation temporarily increases the gap-junction distance between vascular endothelial cells, cause vessel distention and invagination [137], as well as separation of the endothelium from the vessel wall [138]. All these events lead to loss of vascular integrity and increase the permeability to circulating drugs or nanotherapeutics.

In vitro studies have reported an increased endothelial cells permeability following exposure to ultrasounds and microbubbles. This was demonstrated using propidium iodide [139], Dil [140], plasmid DNA [141], fluorescently labelled siRNA [142] and FITC-dextrans with different molecular weight [143]. This latter study reports that endothelial endocytosis, for large molecules, as well as pores formations, for low molecular weight dextrans, were implied in the uptake mechanism. The increased intracellular $\mathrm{H}_{2} \mathrm{O}_{2}$ level quantified in primary endothelial cells after exposure to ultrasounds associated with microbubbles was shown to be involved in the transient increased membrane permeability to ions such as $\mathrm{Ca}^{2+}[144]$. 
In vivo, it was demonstrated in hepatomas models that Evans blue injection followed by

2 ultrasound-targeted microbubble destruction resulted in about fivefold higher Evans blue amount in the target tumours compared with the control ones, underlining an increased capillary permeability [145]. A 13-fold augmentation of endothelial uptake of vascular endothelial growth factor (VEGF) in the myocardium was demonstrated with ultrasounds associated with microbubbles, while ultrasounds alone led to an 8-fold increase, indicating an advantage in associating ultrasound and microbubbles for drug delivery [146].

Several animals' studies using ultrasound and microbubbles to deliver drugs for cancer therapy have demonstrated excellent results. A study using rat liver cancer model examined the biodistribution and tumour delivery of doxorubicin-loaded microbubbles sensitive to ultrasounds and found a significantly higher doxorubicin concentration in the tumours (7-fold) in doxorubicin-loaded microbubbles compared to the administration of free doxorubicin [147]. In a mice breast cancer model, paclitaxel-loaded liposome microbubbles were used to increase drug accumulation in tumours. The drug concentration was up to 4.31-fold higher in tumours compared to the groups without liposomesmicrobubble or ultrasounds [148]. Many other types of cancer have been successfully treated in vivo with ultrasound exposure combine to drug-loaded microbubbles [149].

With respect to the topic at hand, which is the delivery of nanotherapeutics favoured with physical approaches, it has to be noted that drug-loaded nanoparticles attached to microbubbles or simultaneously injected have been explored in sonoporation-mediated drug delivery in cancer. Thus, biodegradable poly (lactic-co-glycolic acid) (PLGA) nanoparticles containing miRNAs administered together with microbubbles in mice bearing human hepatocellular carcinoma xenografts showed a significant therapeutic effect after single treatment since sonoporation significantly increased miRNA delivery by 5-9 fold compared to control conditions [150]. Indeed, sonoporation caused leakage of miRNA-loaded PLGA nanoparticles into the extravascular compartment. Once they crossed the blood 
vessel, PLGA nanoparticles were then endocytosed by tumour cells to release their loading over 15 days [151].

Recently Han et al. proposed to design a nanocomplex responsive to ultrasounds composed of siRNA nanoparticles and paclitaxel-loaded microbubbles [152]. With this two-in-one nanocomplex, focused ultrasound-enabled microbubbles to induce vascular permeability via sonoporation effect, enhancing penetration/accumulation of nanotherapeutics at tumour sites, but also loosening the dense ECM structure. Indeed, the strong acoustic energy subsequently delivered on-site by focused ultrasounds led to protein breakdown [102].

In conclusion, sonoporation which consists in acoustical driven gas microbubbles is a non-invasive and cost-effective strategy that permits a controlled and localised release of nanomedicine to the treated areas, a higher drug accumulation in the tumour while it minimises systemic doses and toxicity. However, it is advisable to remain careful since little is known about consecutive hyperthermia and cellular/tissue response in aftermath of sonoporation.

\subsection{Electroporation}

In a medical point of view, external electric fields present numerous applications and notably in cancer treatment [153]. They can be applied as a standalone therapy to ablate tumours in areas where surgical resection is impossible, to promote cell fusion, or to increase the intracellular penetration of therapeutic molecules such as plasmid DNA of cytotoxic drugs by creating defects in cell membranes. When associated with electro-responsive drug delivery systems, they can also trigger a controlled drug release. In this section, a focus will be made on electric field application leading to electropermeabilization of cells, also named electroporation. Depending on the electric parameters applied, cells can undergo irreversible electroporation (IRE) which is a non-thermal local ablative method for tumours particularly for those close to large blood vessels, or reversible electroporation, widely used for drugs and nucleic acids delivery [154]. In the case of reversible electroporation, plasma 
membrane regains its integrity few minutes after the end of the electric pulse's application but during the permeabilised state, therapeutic molecules can massively enter within the cells. Electrochemotherapy (ECT) is an emergent anti-tumour strategy which consists in associating cytotoxic drug injection with the application of calibrated electric field pulses delivered locally at the tumour site [155]. ECT, which potentiates the cytotoxic effect of drugs by application of external electric field, is currently used in more than 150 clinics throughout Europe.

It has been shown in vivo in normal tissue and tumours that the electrical component of electroporation drastically and transiently reduced the blood flow of treated tissue, this phenomenon is called 'vascular lock' [156-159]. These experimental observations are supported by clinical studies demonstrating the rapid cessation of haemorrhagic melanoma nodule bleeding immediately after electroporation [160]. The first study, using albumin-(Gd-DTPA) contrast-enhanced MRI, has demonstrated 30 min after application of electric pulses that tumour blood volume was reduced from $20 \pm 8 \%$ in control condition (non-exposed to electric field) to $0 \pm 3 \%$ in electroporated ones [157]. This disturbance of the blood flow in the vessels irrigating the tumour is instantaneous after the application of the electric field but reversible. Indeed, blood perfusion restarts about 15 minutes later and its recovery takes place within 48 hours [159]. Besides, mathematical model has demonstrated that endothelial cells lining tumour blood vessels were exposed to the electric field, increasing their permeability. Changes in the shape of endothelial cells were observed 1 hour after the application of electric pulses. The endothelial cells were rounded and swollen, and lumens of blood vessels were narrowed. In addition, within an hour of electroporation, red blood cells accumulated in the treated area and vascular changes contributed to increased infiltration of immune cells [161]. Remodelling of cell junctions (CD31 or PECAM) following electroporation has been confirmed in vivo and associated with permeability of blood vessels to dextran $70 \mathrm{kDa}$ [162]. Indeed, this disturbance of the blood flow induced by electroporation is associated with an increase in tumour vascular permeability in vivo [163165]. In addition, in vitro electroporation of endothelial cells (HUVECs) monolayer has shown the induction of cytoskeleton disturbances (actin filaments and microtubules) as well as an immediate loss 
of intercellular junctions such as VE-Cadherin, increasing the permeability of a model of endothelium [166]. The effects observed with electroporation alone are less dramatic than in the presence of an anticancer agent in electrochemotherapy [167].

All these preclinical and clinical data indicate that electric field applied in electrochemotherapy significantly affects vascular permeability, which suggests that this physical approach could be beneficial to improve nanotherapeutic delivery. To confirm the benefit of electroporation in nanotherapeutics delivery, we can cite a recent article that states that electroporation increased the transport of sorafenib nanoparticles stabilised by a dye (SFB-IR783) through the vascular system, extracellular space and cell membrane [168]. According to the authors, in vitro electroporation increased the permeability of endothelial cell monolayers to these nanoparticles and improved their diffusion through the extracellular space of spheroids. In an HCT-116 in vivo tumour model, increased penetration of nanoparticles into the tumour was linked to the electropermeabilisation of tumour cells but was mediated mainly by changes in vascular permeability and extracellular diffusion. The increased accumulation of nanotherapeutics within the tumor after electroporation resulted in a more efficient antitumor effect than the nanotherapeutics or electroporation alone (Figure 6).

A

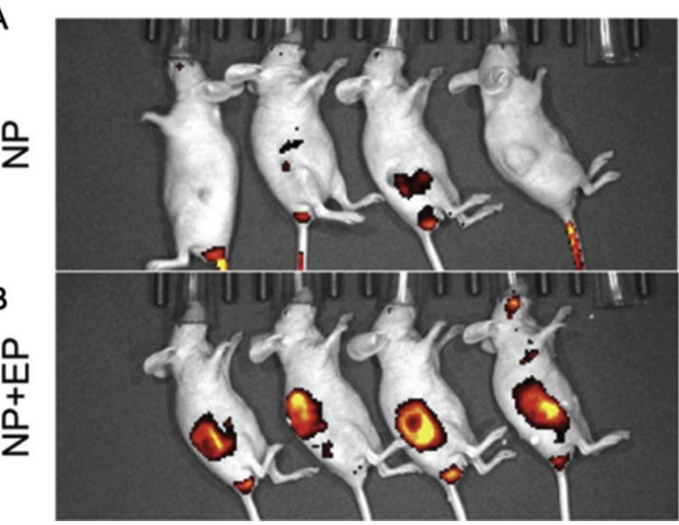

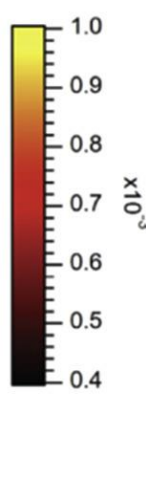

C

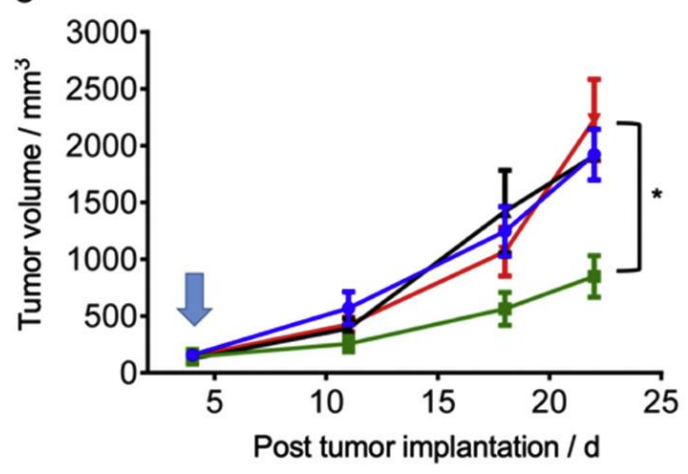

Figure 6: Anti-tumor effect of combined therapy with electroporation (EP) and SFB-IR783. Treatment with EP increased the uptake of SFB-IR783 (B) when compared with mice given the nanoparticle without EP (A). The combined treatment with EP and SFB-IR783 retarded tumor volume in comparison with treatment with EP alone, treatment with SFB-IR783 alone, or control (C). $\square$ EP + NP, $\mathbf{\Delta}$ Control, $\nabla$ EP, • NP. Adapted from [168], Copyright (2020), with permission from Elsevier. 
Interestingly, clinicians and patients reported a scar-free functional healing of the sites treated by

2 electrochemotherapy $[169,170]$, suggesting that electric components of electroporation are involved

3 in the matrix remodelling. However, no study has yet elucidated the effects of reversible electroporation on extracellular matrix components and remodelling enzymes. The only evidence available comes from experiments with irreversible electroporation, reporting the complete recovery of extracellular matrix architecture after treatment [171,172]. However, the extracellular matrix analyses were usually carried out several weeks or months after the end of the treatment, so, early effects of electric fields on ECM are still lacking.

In conclusion, electroporation presents an important potential to increase nanotherapeutics diffusion towards the tumour by simultaneously inducing cells permeabilization (including endothelial, stromal and tumour cells), vascular permeability and probably matrix remodelling.

\subsection{Cold Atmospheric Plasma}

Plasma, known as the fourth state of matter, can be generated by coupling energy to a gas chamber to induce gas ionisation and generate ionised nonthermal gas mixture composed of various ROS, reactive nitrogen species (RNS) and UV photons. Cold atmospheric plasma (CAP) raises an interest in medicine [173], especially in cancer therapy due to the cytotoxic effects arising from the high ROS levels. As plasma medicine is an emerging field, little is known about the effects of plasma on tumour microenvironment. A recent review discusses the effects of cold atmospheric plasma on cells and ECM in tumour context environment [174].

Although several studies show the interest of cold plasma in promoting angiogenesis, particularly in the context of wound healing $[175,176]$, as far as we know there is no study on vascular effects of plasma exposure, particularly in terms of vascular permeability or blood flow modulation. 
It is known that ROS induce ECM remodelling by different mechanisms as an increased expression of MMP-2,-7,-9 and degradation of glycosaminoglycans by hyaluronidase and heparinase [177]. Because cold plasma generates a wide variety of ROS and RNS, it is of prime important to study ECM remodelling after exposure to plasma. Using circular dichroism on bovine type I collagen exposed to cold helium-plasma, Keyvani et al. revealed alterations in the helical structure of dissolved collagen over time, such as oxidation of many structural residues and denaturation although the secondary structure was not damaged [178]. Thus, they demonstrated that the structure of collagen treated with cold atmospheric plasma undergoes oxidation and denaturation. Circular dichroism spectra indicated that $68 \%$ of bovine type I collagen helix structures were denatured after a $30 \mathrm{~s}$ nonthermal argon plasma treatment, which revealed to be very effective in loosening collagen structures [179]. On the contrary, another study analysing the physical structure of the bovine type I collagen by differential scanning calorimetry (DSC) and Fourier transform infrared spectroscopy to check the integrity of the triple helical domain revealed that corona ambient air plasma jet stabilised the collagen structure without altering the triple helical structure [180]. As for many therapeutic strategies, cold atmospheric plasma effects revealed to be dose-dependent [181,182]. For example, Shi et al. suggested that low doses of plasma enhance fibroblasts viability and collagen synthesis while high doses can inhibit them [183]. It seems therefore that plasma has dual effects. Thus, it makes it possible to tune cell fate through modulating the plasma dose for both research and therapeutic purposes but it also appears critical to first tune the parameters according to the desired therapeutic outcome before the application in order to prevent deleterious side effects.

Some rare authors proposed to apply plasma in order to enhance drug delivery. Thus, Vijayarangan et al. demonstrated in vitro on HeLa cells that exposure to a helium plasma jet induced a plasma membrane permeabilization which appeared to be dependent on endocytosis [184]. Using propidium iodide and fluorescent dextrans as permeabilization probes, they proved that plasma was efficient in improving drug delivery at cell scale. Plasma gene transfection also appeared to be efficient as demonstrated by Jinno's work [185]. Zhu et al. demonstrated that 5-FU loaded poly (lactic-co- 
1 glycolic acid) nanoparticles together with cold atmospheric plasma presented a synergistic inhibition

2 of metastatic breast cancer cells (MDA-MB-231) growth when compared to each treatment separately

3 [186]. More interestingly, in this study plasma was found to facilitate drug-loaded nanoparticles uptake

4 by tumour cells, as well as down-regulation of MMP-2 and-9 gene expression. Kim et al. showed in

5 vitro that plasma associated with antibody-conjugated gold nanoparticles led to a near five-fold increase in G361 melanoma cell death when compared to plasma alone [187]. It appears from all these studies that a combined effect between plasma components and reactive species generated is necessary to ensure plasma drug delivery.

Finally, it is curious and interesting to note that cold atmospheric plasma has been employed together with nanotherapeutics (protoporphyrin IX-loaded polymersomes) as an alternative light source in order to kill melanoma cells by photodynamic therapy [188].

\section{Perspectives}

In this review, we have gathered evidence showing that physical approaches, namely radiotherapy, hyperthermia, photodynamic therapy, sonoporation, electroporation and exposure to cold atmospheric plasma, can efficiently remodel tumour microenvironment through modulation of vascular permeability and remodelling of the extracellular matrix, thus enabling an improved delivery of nanotherapeutics to the tumour (Figure 7), (Table 2). 


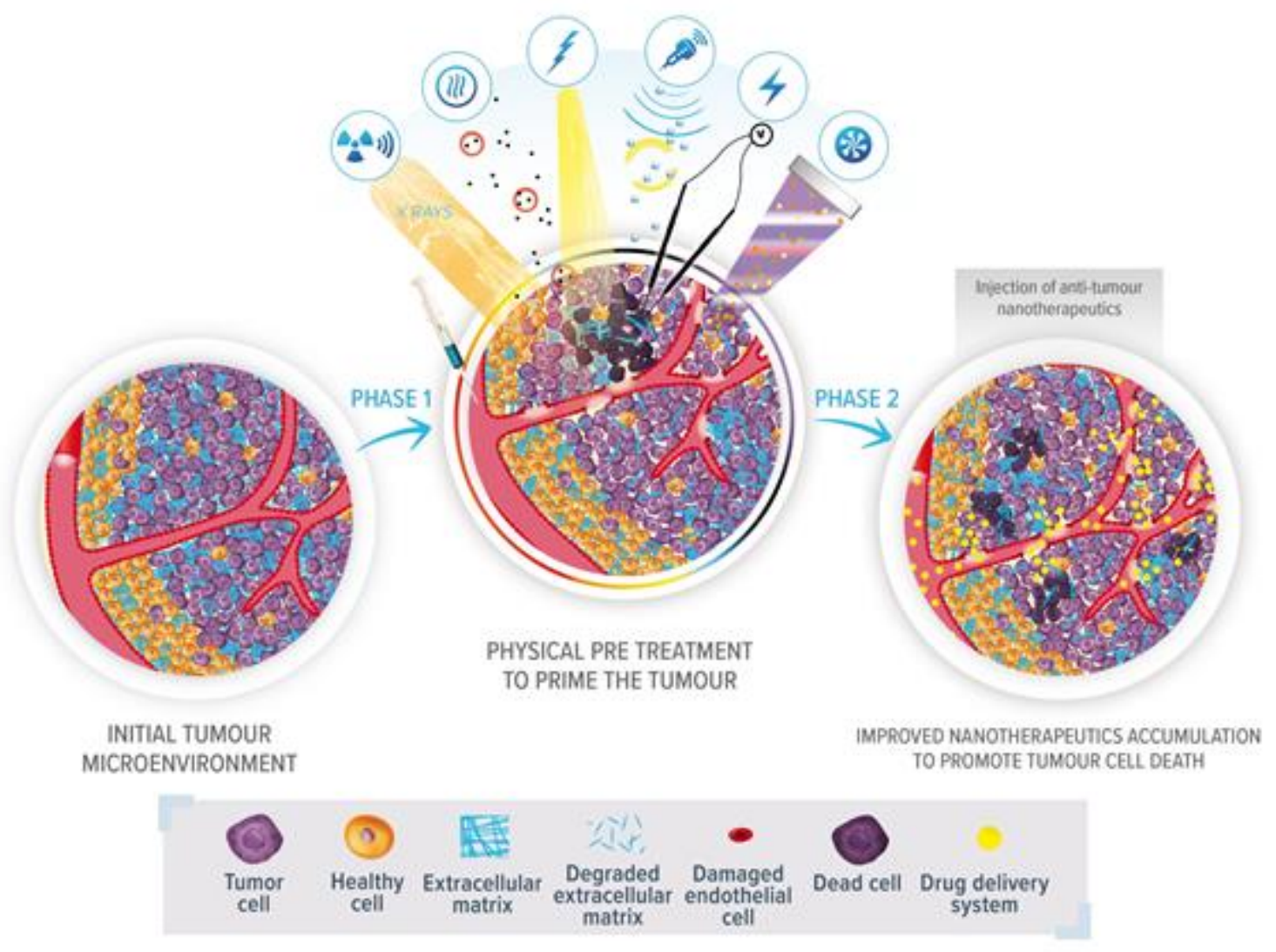

1

Figure 7: Schematic view of the effects on vasculature and extracellular matrix degradation by different physical approaches in perspective of multimodal anticancer therapy. Priming the tumor thanks to physical approaches offers a therapeutic window to improve nanotherapeutics antitumor efficiency.

5

6

7 Table 2. Summary of the effects (described in the text) of the different physical approaches on vascular 8 and matrix remodeling for improved delivery of nanotherapeutics. Reviews are not included in this 9 table, only original articles. ECM: extracellular matrix. MB: microbubbles. 
Endothelial damages and vascular permeability increase $[32,33,35,41]$ (in vivo)

\section{Radiotherapy}

Decreased interstitial fluid pressure $[39-41,67]$ (in vivo/ clinics)

Modulation of blood flow rate: Increase [80], no increase [83], impairment [76,84] (in vivo)

Deformation of blood vessels leading to coagulation [85] and haemorrhage $[83,86,87$ ] (in vivo)

Increased vascular permeability to different molecules or nanotherapeutics [77-81] (in vivo)

\section{Decrease of ECM stiffness [86] (in vivo)}

Denaturation/melting of collagens $[96,101,104]$ (in vivo), [93-95,97] (in vitro)

MMPs mRNA: upregulation of MMP-1,-3 [126] (in vitro), MMP-1 [128] (in vitro)

MMPs proteins: Increased expression of MMP-1, MMP-9, and TGF- $\beta 3$ production [120] (clinics)

Endothelial damages and vascular permeability increase [108] (in vivo)

Increase of tumor interstitial fluid pressure [109] (in vivo)

Decreased tumour interstitial fluid pressure [116] (in vivo)

Endothelial cell alterations [110-112] (in vitro)
Photodynamic therapy
MMPs mRNA: upregulation of MMP-2,-3,-9,14

vivo), MMP-9 [47] (clinics), MMP-1,-2,-9

MMPs activity: increased production of MMP-2 $[46,49,50]$ (in vitro), $[47,48]$ (clinics), PAI-I $[56]$ (clinics) nanoparticles [41], Dox/or gemcitabine -HPMA [65], liposomal doxorubicin (Caelyx) [66], liposomal doxorubicin (Doxil) [67] (in vivo)
MMPs and EMMPRIN [125] (in vivo), MMP-1,-3 126] (in vitro). Decreased expression of MMP-1,2,-9 [121] (in vitro) and TIMP-1 [125] (in vivo)

Hypericin photosensitizer presents affinity to collagen [122] (in vitro)

ECM mRNA: downregulation of type I collagen [126], TGF-b1, COL1A2, COL3A1 [128] (in vitro)

ECM proteins: Increased production of collagen I, collagen III and elastin [120] (clinics), [119] (in vivo), [121] (in vitro). Degradation of collagens [123] (in vitro) and [124] (ex vivo)
Increased delivery/cell uptake of doxorubicin loaded in thermosensitive liposomes [83], elastin-like peptides [89], iron oxide nanocubes [90], nanospheres [101], DOX-loaded in thermoresponsive PNIPAM liposomes [96] or mertansine-lipoproteins [104] (in vivo), iron oxide nanoparticles [97] (in vitro)
Improved delivery and efficacy of oncolytic vaccinia after HPPH-PDT [113] (in vivo)

ncreased tumor uptake of liposomal doxorubicin (Doxil) [114], liposomal doxorubicin (Liporubicin) $[115,116]$ or liposomal daunorubicin (DaunoXome) [117] and antitumor activity (in vivo) 
Loss of vascular integrity and increased

permeability [136-138,145] (in vivo)

Increased endothelial cells permeability to propidium iodide [139], Dil [140], plasmid DNA

[141], fluorescently labelled siRNA [142] and

FITC-dextrans [143] (in vitro)

Increased intracellular $\mathrm{H}_{2} \mathrm{O}_{2}$ level in endothelial cells [144] (in vitro)

Drastically and transient reduction in the blood

flow of treated tissue 'vascular lock' and vascular

permeability [156-160] (in vivo/ clinics)

Endothelial cell alterations [161-165] (in vivo)

and $[166,167]$ (in vitro)

Cold atmospheric

plasma
Promotes angiogenesis [175,176] (in vitro/in vivo)
Loosening of dense ECM structure [102] (in vivo) nanoparticles [102], doxorubicin-loaded MB [147], paclitaxel-loaded liposome MB [148] and others drug-loaded MB [149] (in vivo)

Improved therapeutic effect when associated with miRNA-loaded PLGA nanoparticles [150] through sustained intratumoral release [151] (in vivo)

Recovery of extracellular matrix architecture after irreversible electroporation treatment $[171,172]$ (in vivo)

Increased transport and accumulation of sorafenib nanoparticles stabilised by a dye (SFBIR783) [168] (in vitro/in vivo)

Alteration of type I collagen [178] [179] (in vitro)

Stabilisation of type I collagen structure [180] (in vitro)

Affect collagen synthesis by fibroblasts depending on doses [183] (in vitro)

Down-regulation of MMP-2 and-9 gene expression in breast cancer cells [186] (in vitro)
Plasma membrane permeabilization to propidium iodide and fluorescent dextrans [184] (in vitro)

Efficient plasma gene transfection [185] (in vitro) Increase uptake of 5-FU loaded poly (lactic-coglycolic acid) nanoparticles [186] or antibodyconjugated gold nanoparticles [187] (in vitro) 
However, some points were not addressed and have to be mentioned. For instance, an innovative approach based on nanomedicine aims to directly target the tumour microenvironment rather than cancer cells, e.g. the extracellular matrix itself [189] (Figure 8), cancer-associated cells such as cancerassociated fibroblasts (CAF) [190] or the physiological microenvironment (hypoxia, acidic environment...) [91]. The main advantage of this approach is that it has the potential to eliminate all types of cells from the tumour microenvironment (cancer cells, cancer-associated fibroblasts and macrophages, etc.). The review published by Raavé et al. provides an interesting overview of drug delivery strategies targeting the tumour extracellular matrix [189]. The current challenge is to trigger the release of the drug once the nanovector is bound to the tumour extracellular matrix. This involves the design of smart vectors sensitive to, for example, certain types of enzymes, $\mathrm{pH}$, heat, light or ultrasound.

Binding of drug delivery system to tumor extracellular matrix

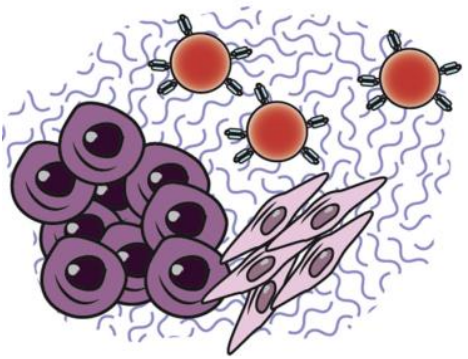

Extracellular drug release

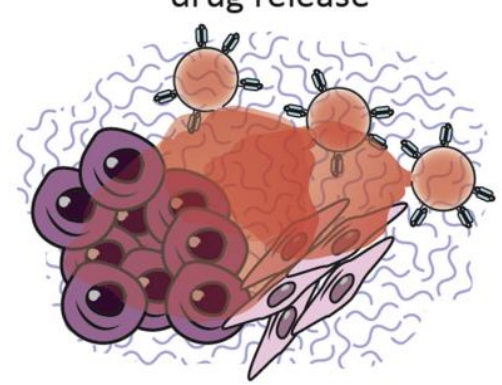

Eradication of both tumor and tumor supportive cells

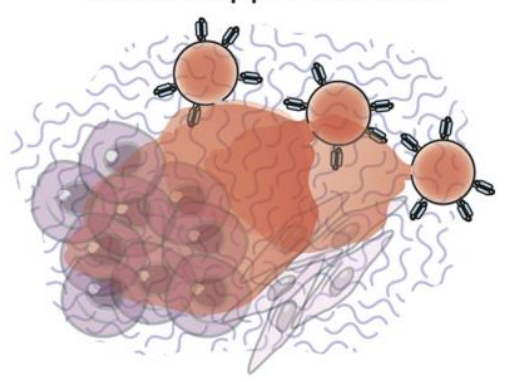

Figure 8: Overview of tumour extracellular matrix targeting by drug delivery systems. Nanotherapeutics target a specific molecule abundantly present in the tumour extracellular matrix. Once bound, the drug is released and diffuses into neighbouring cells (tumour cells, cancer-associated fibroblasts, cancer-associated macrophages, etc.). From $[189,191]$ under the Creative Commons Attribution License (CC BY 4.0).

Targeting cancer-associated fibroblasts (CAF) in order to decrease collagen content within the tumour microenvironment is another strategy to explore, as in this war against cancer, the enhancement of nanomedicine delivery at the tumour site is the goal to be achieved. Thus, Li et al. encapsulated ZnF16Pc, a photosensitiser, into ferritin nanocages decorated on their surface with a single chain viable fragment (scFv) sequence (scFv-Z@FRT) specific to fibroblast activation protein (FAP), upregulated in CAF [192]. Significantly reduced levels of collagens were observed together with tumour accumulation of serum albumin, $10 \mathrm{~nm}$ and $50 \mathrm{~nm}$ quantum dots, increased respectively by 2- 
, 3.5-, and 18-fold after scFv-Z@FRT mediated PDT. These results suggest that targeting CAF is an efficient and safe method to enhance the delivery of nanoparticles to tumours by breaking down the ECM barrier with minimal side effects.

The potential of these physical strategies to activate the immune system to fight against the tumour is out of the scope of this review but deserves to be mentioned because increasing evidence shows their interest in this field.

Besides, it should be noted that the physicochemical properties of free or formulated drugs (molecular weight, form, charge and aqueous solubility) determine their capacity/rate of diffusion through the tissue [20]. Therefore, water-soluble drugs are more easily distributed within the extracellular matrix, in particular thanks to highly hydrated glycosaminoglycans while hydrophobic drugs penetrate lipid membranes and can therefore be transported through cells. Thus, an optimised formulation can increase drug penetration within tumour tissue and aid the development of more effective anticancer drugs by improving their therapeutic index. For instance, doxorubicin encapsulated in liposomes effectively alter the pharmacokinetics of the free drug and take advantage of the permeability of tumour blood vessels to liposomal particles [193]. Dreher et al. have shown that using dextran covalently linked to a fluorophore as a model macromolecular drug carrier led to a shallower penetration into the tumour interstitium with increasing molecular weight [194]. Even if nano-sized drug delivery systems generally have low molecular weight, many of them show significant binding to plasma proteins, which leads them to behave, functionally, like macromolecules [195]. This protein corona forming on most nanoparticles confers to them a new biological identity [196] that affects their interactions with the biological environment and determines biological events such as immune response, biodistribution, cellular uptake... Thus, the protein corona has to be taken into consideration when designing drug delivery anti-tumour strategies.

Finally, in a promising way, these physical approaches (focused ultrasound, magnetic heating of nanoparticles, radiation therapy, electric fields, laser therapy) were shown to be efficient to disrupt 
blood-brain barrier (BBB) and improve nanoparticle delivery at cerebral site [197]. For example, transient (for hours) BBB disruption has been accomplished using focused ultrasound exposure combined with microbubbles, allowing extravasation of therapeutic agents such as liposomal doxorubicin while causing little or no damage to brain tissue [198]. High-frequency electroporation was demonstrated to be efficient in disrupting BBB for less than 96h, with minimal muscle contractions and minimal cell death attributed to the electric field [199]. However, clinical devices and methods need to be developed further and the bench-to-bedside translation demonstrated.

\section{Expert Opinion}

The advances in physical treatments of tumour microenvironment we have described here above have underpinned the extension of local and specific treatments towards cancer cells. Full exploitation of these advancements allows to maximize chance of selectively kill tumour cells, while minimising effects on normal tissue in and around the targeted area. Their concomitant use with chemotherapeutic agents increases therapy performances while reducing severe side effects of these therapeutics. The possible use of an external technique to control in space and time the treatment is a great response to practitioners and patients. The ultimate goal to reach in cancer treatment would be one therapy relying only on external triggering. A great step forward as, historically, only invasive techniques like excision, ablation and then toxic therapeutics where employed, affecting, sometimes dramatically, patients' compliance. This review, thus, focuses on recent progress achieved in physical methods whose trigger has proven their effectiveness in cancer care performance. However and retrospectively, this plethora of available physical technic led to the same consequences: external treatment has very often a strong impact on the tumour but irreversible effects are only obtained when high intensity parameters are used, causing to inevitable deleterious side effects. In this particular context, maximising tumour cells destruction may need to be sacrificed in favour of less deleterious strategies. It goes without saying that it is quite natural that the world of research has 
moved towards the joint use of chemo- and physico-therapy in a multimodal therapy in order to improve clinical outcomes for patients affected by cancer as well as their quality of life.

Combining physical strategies with drug-loaded nanotherapeutics in patients present a high translational impact since it may allow for both chemical and physical dose reductions enabling further sparing of healthy tissues. However, some limitations may occasionally appear in the use of these physical approaches, in particular due to tumour architecture or location. For example, deep-seated tumors can be hard to treat due to low tissue penetration of visible light.

One of the major strengths of physical approaches to improve the delivery of nanotherapeutics is the local and transient aspect of their effects. A typical example is the membrane permeabilization that occurs when electroporation is used as a drug delivery tool. This permeabilization is transient and fades within minutes after ending external electric field application. This transient aspect illustrates the existence of a therapeutic window, underling the major importance of time interval between completion of physical triggering and administration of nanotherapeutics. Of course, for each clinical application envisaged, the co-administration protocol must be clearly validated experimentally. However, most of the experimental data obtained with hyperthermia, PDT, electroporation or sonoporation indicate that since the physical conditions applied are mild, the injection of nanotherapeutics should be considered within minutes.

Notably, the physical therapies presented in this review (radiotherapy, hyperthermia, photodynamic therapy, sonoporation, electroporation and cold atmospheric plasma) are already clinically approved, ensuring, thus, potentially fast clinical translation when combined with FDA nanotherapeutics. We emphasise here the importance of using physical therapies as a neoadjuvant to enhance the delivery of nanotherapeutics to the tumour and to adapt therapeutic protocols according to the patient/tumour specificity. Thus, this review illustrates that for each type of cancer, different chem/physical therapies can be proposed jointly, delivering a fully personalised therapy. 
2 perspectives, not only to reduce/destroy the tumour, but also to restrict its resistance to therapies,

3 activate the immune response and reduce the risk of recurrence. The clinical evidence obtained for years has proven the effectiveness of the combination of radiotherapy with drugs and/or nanotherapeutics. These examples, abundantly described in the literature, allow us to view emerging physical stimuli optimistically in the fight against cancer.

To achieve applicable clinical protocols, cellular and tissue biological mechanisms impacted in multimodal therapies must first be widely studied and understood from a pre-clinical point of view before considering their translation to the clinical level. In addition, to ensure their broad and correct use in the clinic, oncologists and other practitioners need to become familiar with these physical techniques, which is typically the case for PDT. Indeed, efficient PDT implies the control of appropriate parameters regarding the choice of the photosensitiser, the wavelength needed, the light intensity, pulses duration... if not well controlled, early and late onset side effects may arise (pain, burns, oedema, itching, desquamation). Thus, the complexity and multi-parametric aspects of multimodal therapies are highlighted in the different parts of the article. The multidisciplinary coordination of professional profiles such as physicists, physicians, chemists and biologists is necessary for treatment optimisation and use. This observation led to a new vision of health care organised around a team of specialists at the service of each patient similar to what is done in the research sector.

From our opinion, except for RT which is already widely used in clinics, all other physical methods presented in this review will be increasingly translated into clinical treatments in the coming years if systematically combined to chemotherapy. Indeed, their ability to increase vascular permeability and ECM degradation was proven. Subsequent penetration of drugs, especially for nanotherapeutics in tumour sites can be expected while increasing increases the chances of success of the therapy as well as patient compliance. In addition, these physical approaches have the invaluable advantage of acting locally, i.e. only on the desired site, as opposed to more aggressive chemotherapy. 
This work was supported by the French Agence Nationale de la recherche under Grant ANR-17-CE19-

0013-01.

Financial and competing interests disclosure

The authors report no conflicts of interest.

8

\section{Acknowledgments}

Lab2screen is acknowledged for English proofreading and editing as well as design creations for graphical abstract, figures 2 and 7 .

\section{References}

Papers of special note have been highlighted as either of interest $(\bullet)$ or of considerable interest $(\bullet \bullet)$ to readers.

[1] Li Y, Wang J, Wientjes MG, et al. Delivery of nanomedicines to extracellular and intracellular compartments of a solid tumor. Adv Drug Deliv Rev. 2012;64:29-39.

[2] Marcucci F, Corti A. How to improve exposure of tumor cells to drugs - Promoter drugs increase tumor uptake and penetration of effector drugs. Adv Drug Deliv Rev. 2012;64:5368.

[3] Carmeliet P, Jain RK. Angiogenesis in cancer and other diseases. nature. 2000;407:249.

[4] Matsumura Y, Maeda H. A New Concept for Macromolecular Therapeutics in Cancer Chemotherapy: Mechanism of Tumoritropic Accumulation of Proteins and the Antitumor Agent Smancs. Cancer Res. 1986;46:6387-6392.

[5] Nichols JW, Bae YH. EPR: Evidence and fallacy. J Controlled Release. 2014;190:451-464. 
[6] Heldin $\mathrm{C}-\mathrm{H}$, Rubin $\mathrm{K}$, Pietras $\mathrm{K}$, et al. High interstitial fluid pressure - an obstacle in cancer therapy. Nat Rev Cancer. 2004;4:806-813.

[7] Ariffin AB, Forde PF, Jahangeer $S$, et al. Releasing Pressure in Tumors: What Do We Know So Far and Where Do We Go from Here? A Review. Cancer Res. 2014;74:2655-2662.

[8] - Ojha T, Pathak V, Shi Y, et al. Pharmacological and physical vessel modulation strategies to improve EPR-mediated drug targeting to tumors. Adv Drug Deliv Rev. 2017;119:44-60.

Overview of pharmacological and physical strategies to normalize, permeabilize, disrupt or promote tumour blood vessels in order to improve drug delivery systems accumulation within tumor.

[9] Dhaliwal A, Zheng G. Improving accessibility of EPR-insensitive tumor phenotypes using EPRadaptive strategies: Designing a new perspective in nanomedicine delivery. Theranostics. 2019;9:8091.

[10] Yang S, Gao H. Nanoparticles for modulating tumor microenvironment to improve drug delivery and tumor therapy. Pharmacol Res. 2017;126:97-108.

[11] Zhou Y, Chen X, Cao J, et al. Overcoming the biological barriers in the tumor microenvironment for improving drug delivery and efficacy. J Mater Chem B. 2020;8:67656781.

[12] Henke E, Nandigama R, Ergün S. Extracellular Matrix in the Tumor Microenvironment and Its Impact on Cancer Therapy. Front Mol Biosci. 2020;6:160.

[13] Paszek MJ, Zahir N, Johnson KR, et al. Tensional homeostasis and the malignant phenotype. Cancer Cell. 2005;8:241-254.

[14] Kauppila S, Stenbäck F, Risteli J, et al. Aberrant type I and type III collagen gene expression in human breast cancer in vivo. J Pathol J Pathol Soc G B Irel. 1998;186:262-268.

[15] Theocharis AD, Skandalis SS, Gialeli C, et al. Extracellular matrix structure. Adv Drug Deliv Rev. 2016;97:4-27.

[16] Lu P, Weaver VM, Werb Z. The extracellular matrix: A dynamic niche in cancer progression. J Cell Biol. 2012;196:395-406.

[17] Netti PA, Berk DA, Swartz MA, et al. Role of extracellular matrix assembly in interstitial transport in solid tumors. Cancer Res. 2000;60:2497-2503.

[18] Jain RK. Normalizing Tumor Microenvironment to Treat Cancer: Bench to Bedside to Biomarkers. J Clin Oncol. 2013;31:2205-2218.

[19] Burgstaller G, Oehrle B, Gerckens M, et al. The instructive extracellular matrix of the lung: basic composition and alterations in chronic lung disease. Eur Respir J. 2017;50:1601805.

[20] $\quad \bullet$ Minchinton Al, Tannock IF. Drug penetration in solid tumours. Nat Rev Cancer. 2006;6:583-592.

Focus on drug distribution visualization within tumours from in vitro to in vivo studies and proposes strategies that might be used to improve drug penetration in order to increase its therapeutic index. 
[21] Brown E, McKee T, diTomaso E, et al. Dynamic imaging of collagen and its modulation in tumors in vivo using second-harmonic generation. Nat Med. 2003;9:796-800.

[22] Dolor A, Szoka FC. Digesting a Path Forward: The Utility of Collagenase Tumor Treatment for Improved Drug Delivery. Mol Pharm. 2018;15:2069-2083.

[23] Chauhan VP, Martin JD, Liu H, et al. Angiotensin inhibition enhances drug delivery and potentiates chemotherapy by decompressing tumour blood vessels. Nat Commun. 2013;4:111.

[24] Barry-Hamilton V, Spangler R, Marshall D, et al. Allosteric inhibition of lysyl oxidase-like-2 impedes the development of a pathologic microenvironment. Nat Med. 2010;16:1009.

[25] Coussens LM, Fingleton B, Matrisian LM. Matrix metalloproteinase inhibitors and cancertrials and tribulations. Science. 2002;295:2387-2392.

[26] Diop-Frimpong B, Chauhan VP, Krane S, et al. Losartan inhibits collagen I synthesis and improves the distribution and efficacy of nanotherapeutics in tumors. Proc Natl Acad Sci. 2011;108:2909-2914.

[27] Khawar IA, Kim JH, Kuh H-J. Improving drug delivery to solid tumors: Priming the tumor microenvironment. J Controlled Release. 2015;201:78-89.

[28] Nakamura Y, Mochida A, Choyke PL, et al. Nanodrug Delivery: Is the Enhanced Permeability and Retention Effect Sufficient for Curing Cancer? Bioconjug Chem. 2016;27:2225-2238.

[29] Wang Y, Gan G, Wang B, et al. Cancer-associated Fibroblasts Promote Irradiated Cancer Cell Recovery Through Autophagy. EBioMedicine. 2017;17:45-56.

[30] Hanahan D, Weinberg RA. Hallmarks of Cancer: The Next Generation. Cell. 2011;144:646674.

[31] Fuks Z, Kolesnick R. Engaging the vascular component of the tumor response. Cancer Cell. 2005;8:89-91.

[32] Potiron VA, Abderrahmani R, Clément-Colmou K, et al. Improved functionality of the vasculature during conventionally fractionated radiation therapy of prostate cancer. PloS One. 2013;8:e84076.

[33] Garcia-Barros M, Paris F, Cordon-Cardo C, et al. Tumor response to radiotherapy regulated by endothelial cell apoptosis. Science. 2003;300:1155-1159.

[34] $\quad \bullet$ DuRoss AN, Neufeld MJ, Rana S, et al. Integrating nanomedicine into clinical radiotherapy regimens. Adv Drug Deliv Rev. 2019;144:35-56.

A review summarizing how nanomedicine and radiotherapy can participate to multimodal strategies

[35] Schwickert HC, Stiskal M, Roberts TP, et al. Contrast-enhanced MR imaging assessment of tumor capillary permeability: Effect of irradiation on delivery of chemotherapy. Radiology. 1996;198:893-898.

[36] Moding EJ, Kastan MB, Kirsch DG. Strategies for optimizing the response of cancer and normal tissues to radiation. Nat Rev Drug Discov. 2013;12:526-542. 
[37] Stapleton S, Jaffray D, Milosevic M. Radiation effects on the tumor microenvironment: Implications for nanomedicine delivery. Adv Drug Deliv Rev. 2017;109:119-130.

[38] Shi J, Kantoff PW, Wooster R, et al. Cancer nanomedicine: progress, challenges and opportunities. Nat Rev Cancer. 2017;17:20.

[39] Roh HD, Boucher $\mathrm{Y}$, Kalnicki S, et al. Interstitial hypertension in carcinoma of uterine cervix in patients: possible correlation with tumor oxygenation and radiation response. Cancer Res. 1991;51:6695-6698.

[40] Znati CA, Rosenstein M, Boucher Y, et al. Effect of radiation on interstitial fluid pressure and oxygenation in a human tumor xenograft. Cancer Res. 1996;56:964-968.

[41] Giustini AJ, Petryk AA, Hoopes PJ. Ionizing radiation increases systemic nanoparticle tumor accumulation. Nanomedicine Nanotechnol Biol Med. 2012;8:818-821.

[42] Moeller BJ, Cao Y, Li CY, et al. Radiation activates HIF-1 to regulate vascular radiosensitivity in tumors: Role of reoxygenation, free radicals, and stress granules. Cancer Cell. 2004;5:429441.

[43] Dewhirst MW, Cao Y, Moeller B. Cycling hypoxia and free radicals regulate angiogenesis and radiotherapy response. Nat Rev Cancer. 2008;8:425-437.

[44] Lee C-G, Heijn M, di Tomaso E, et al. Anti-vascular endothelial growth factor treatment augments tumor radiation response under normoxic or hypoxic conditions. Cancer Res. 2000;60:5565-5570.

[45] Tachiiri S, Katagiri T, Tsunoda T, et al. Analysis of gene-expression profiles after gamma irradiation of normal human fibroblasts. Int J Radiat Oncol Biol Phys. 2006;64:272-279.

[46] Qian L-W, Mizumoto K, Urashima T, et al. Radiation-induced increase in invasive potential of human pancreatic cancer cells and its blockade by a matrix metalloproteinase inhibitor, CGS27023. Clin Cancer Res. 2002;8:1223-1227.

[47] Kumar A, Collins HM, Scholefield JH, et al. Increased type-IV collagenase (MMP-2 and MMP9) activity following preoperative radiotherapy in rectal cancer. Br J Cancer. 2000;82:960965.

[48] Angenete $E$, Öresland $T$, Falk $P$, et al. Preoperative radiotherapy and extracellular matrix remodeling in rectal mucosa and tumour matrix metalloproteinases and plasminogen components. Acta Oncol. 2009;48:1144-1151.

[49] Chetty C, Bhoopathi P, Rao JS, et al. Inhibition of matrix metalloproteinase-2 enhances radiosensitivity by abrogating radiation-induced FoxM1-mediated G2/M arrest in A549 lung cancer cells. Int J Cancer. 2009;124:2468-2477.

[50] Paquette $\mathrm{B}$, Baptiste $\mathrm{C}$, Therriault $\mathrm{H}$, et al. In vitro irradiation of basement membrane enhances the invasiveness of breast cancer cells. Br J Cancer. 2007;97:1505-1512.

[51] Speake WJ, Dean RA, Kumar A, et al. Radiation induced MMP expression from rectal cancer is short lived but contributes to in vitro invasion. Eur J Surg Oncol EJSO. 2005;31:869-874. 
[52] Shankar A, Kumar S, Iskander A, et al. Subcurative radiation significantly increases cell proliferation, invasion, and migration of primary glioblastoma multiforme in vivo. Chin J Cancer. 2014;33:148.

[53] Ager El, Kozin SV, Kirkpatrick ND, et al. Blockade of MMP14 activity in murine breast carcinomas: implications for macrophages, vessels, and radiotherapy. JNCI J Natl Cancer Inst. 2015;107.

[54] Strup-Perrot C, Vozenin-Brotons M-C, Vandamme M, et al. Expression and activation of MMP $-2,-3,-9,-14$ are induced in rat colon after abdominal X-irradiation. Scand J Gastroenterol. 2006;41:60-70.

[55] Angenete E, Langenskiöld M, Falk P, et al. Matrix metalloproteinases in rectal mucosa, tumour and plasma: response after preoperative irradiation. Int J Colorectal Dis. 2007;22:667-674.

[56] Angenete E, Langenskiöld M, Palmgren I, et al. uPA and PAI-1 in rectal cancer-relationship to radiotherapy and clinical outcome. J Surg Res. 2009;153:46-53.

[57] Kagan HM, Li W. Lysyl oxidase: properties, specificity, and biological roles inside and outside of the cell. J Cell Biochem. 2003;88:660-672.

[58] Erler JT, Giaccia AJ. Lysyl Oxidase Mediates Hypoxic Control of Metastasis. Cancer Res. 2006;66:10238-10241.

[59] Egeblad M, Rasch MG, Weaver VM. Dynamic interplay between the collagen scaffold and tumor evolution. Curr Opin Cell Biol. 2010;22:697-706.

[60] Shen CJ, Sharma A, Vuong D-V, et al. Ionizing radiation induces tumor cell lysyl oxidase secretion. BMC Cancer. 2014;14:532.

[61] Xie P, Yu H, Wang F, et al. Inhibition of LOXL2 Enhances the Radiosensitivity of CastrationResistant Prostate Cancer Cells Associated with the Reversal of the EMT Process. Catala A, editor. BioMed Res Int. 2019;2019:4012590.

[62] Chen HH, Kuo MT. Improving radiotherapy in cancer treatment: promises and challenges. Oncotarget. 2017;8:62742.

[63] Li J, Shang W, Li Y, et al. Advanced nanomaterials targeting hypoxia to enhance radiotherapy. Int J Nanomedicine. 2018;13:5925.

[64] Lammers T, Peschke P, Kühnlein R, et al. Effect of radiotherapy and hyperthermia on the tumor accumulation of HPMA copolymer-based drug delivery systems. J Controlled Release. 2007;117:333-341.

[65] Lammers T, Subr V, Peschke $P$, et al. Image-guided and passively tumour-targeted polymeric nanomedicines for radiochemotherapy. Br J Cancer. 2008;99:900-910.

[66] Davies C de L, Lundstrøm LM, Frengen J, et al. Radiation improves the distribution and uptake of liposomal doxorubicin (caelyx) in human osteosarcoma xenografts. Cancer Res. 2004;64:547-553. 
[67] $\quad \because$ Stapleton S, Dunne M, Milosevic M, et al. Radiation and heat improve the delivery and efficacy of Nanotherapeutics by modulating Intratumoral fluid dynamics. ACS Nano. 2018;12:7583-7600.

\section{Effect of the IFP on the tumor response to RT}

[68] Miller MA, Chandra R, Cuccarese MF, et al. Radiation therapy primes tumors for nanotherapeutic delivery via macrophage-mediated vascular bursts. Sci Transl Med. 2017;9.

[69] Koukourakis MI, Koukouraki S, Giatromanolaki A, et al. Liposomal doxorubicin and conventionally fractionated radiotherapy in the treatment of locally advanced non-small-cell lung cancer and head and neck cancer. J Clin Oncol. 1999;17:3512-3521.

[70] Rosenthal DI, Yom SS, Liu L, et al. A Phase I study of SPI-077 (Stealth ${ }^{\circledR}$ liposomal cisplatin) concurrent with radiation therapy for locally advanced head and neck cancer. Invest New Drugs. 2002;20:343-349.

[71] Dipetrillo T, Milas L, Evans D, et al. Paclitaxel poliglumex (PPX-Xyotax) and concurrent radiation for esophageal and gastric cancer: a phase I study. Am J Clin Oncol. 2006;29:376379.

[72] Zee J van der, Vujaskovic Z, Kondo M, et al. The Kadota Fund International Forum 2004Clinical group consensus*. Int J Hyperthermia. 2008;24:111-122.

[73] Koning GA, Eggermont AMM, Lindner LH, et al. Hyperthermia and Thermosensitive Liposomes for Improved Delivery of Chemotherapeutic Drugs to Solid Tumors. Pharm Res. 2010;27:1750-1754.

[74] Hu Q, Huang Z, Duan Y, et al. Reprogramming Tumor Microenvironment with Photothermal Therapy. Bioconjug Chem. 2020;31:1268-1278.

[75] Datta NR, Kok HP, Crezee H, et al. Integrating Loco-Regional Hyperthermia Into the Current Oncology Practice: SWOT and TOWS Analyses. Front Oncol. 2020;10:819.

[76] Vaupel PW, Kelleher DK. Pathophysiological and vascular characteristics of tumours and their importance for hyperthermia: heterogeneity is the key issue. Int J Hyperthermia. 2010;26:211-223.

[77] Kirui DK, Koay EJ, Guo X, et al. Tumor vascular permeabilization using localized mild hyperthermia to improve macromolecule transport. Nanomedicine Nanotechnol Biol Med. 2014;10:1487-1496.

[78] Melancon MP, Elliott AM, Shetty A, et al. Near-infrared light modulated photothermal effect increases vascular perfusion and enhances polymeric drug delivery. J Controlled Release. 2011;156:265-272.

[79] Lapin NA, Krzykawska-Serda M, Dilliard S, et al. The effects of non-invasive radiofrequency electric field hyperthermia on biotransport and biodistribution of fluorescent [60]fullerene derivative in a murine orthotopic model of breast adenocarcinoma. J Controlled Release. 2017;260:92-99.

[80] Li L, ten Hagen TLM, Bolkestein M, et al. Improved intratumoral nanoparticle extravasation and penetration by mild hyperthermia. J Controlled Release. 2013;167:130-137. 
[81] Gao H, Bi Y, Chen J, et al. Near-Infrared Light-Triggered Switchable Nanoparticles for Targeted Chemo/Photothermal Cancer Therapy. ACS Appl Mater Interfaces. 2016;8:1510315112.

[82] Baronzio GF, Galante F, Gramaglia A, et al. Tumor microcirculation and its significance in therapy: possible role of omega-3 fatty acids as rheological modifiers. Med Hypotheses. 1998;50:175-182.

[83] Fite BZ, Kheirolomoom A, Foiret JL, et al. Dynamic contrast enhanced MRI detects changes in vascular transport rate constants following treatment with thermally-sensitive liposomal doxorubicin. J Controlled Release. 2017;256:203-213.

[84] Song CW, Park HJ, Lee CK, et al. Implications of increased tumor blood flow and oxygenation caused by mild temperature hyperthermia in tumor treatment. Int J Hyperthermia. 2005;21:761-767.

[85] Corr SJ, Shamsudeen S, Vergara LA, et al. A New Imaging Platform for Visualizing Biological Effects of Non-Invasive Radiofrequency Electric-Field Cancer Hyperthermia. Muñoz-Barrutia A, editor. PLOS ONE. 2015;10:e0136382.

[86] Marangon I, Silva AAK, Guilbert T, et al. Tumor Stiffening, a Key Determinant of Tumor Progression, is Reversed by Nanomaterial-Induced Photothermal Therapy. Theranostics. 2017;7:329-343.

[87] - Kolosnjaj-Tabi J, Marangon I, Nicolas-Boluda A, et al. Nanoparticle-based hyperthermia, a local treatment modulating the tumor extracellular matrix. Pharmacol Res. 2017;126:123137.

A review summarizing tumor micro-environment and how hyperthermia can modulate ECM

[88] Yudina A, Moonen C. Ultrasound-induced cell permeabilisation and hyperthermia: Strategies for local delivery of compounds with intracellular mode of action. Int J Hyperthermia. 2012;28:311-319.

[89] Ryu JS, Raucher D. Elastin-like polypeptides: The influence of its molecular weight on local hyperthermia-induced tumor accumulation. Eur J Pharm Biopharm. 2014;88:382-389.

[90] Kolosnjaj-Tabi J, Di Corato R, Lartigue L, et al. Heat-Generating Iron Oxide Nanocubes: Subtle "Destructurators" of the Tumoral Microenvironment. ACS Nano. 2014;8:4268-4283.

[91] Thakkar S, Sharma D, Kalia K, et al. Tumor microenvironment targeted nanotherapeutics for cancer therapy and diagnosis: A review. Acta Biomater. 2020;101:43-68.

[92] Lokerse WJM, Bolkestein M, Dalm SU, et al. Comparing the therapeutic potential of thermosensitive liposomes and hyperthermia in two distinct subtypes of breast cancer. J Controlled Release. 2017;258:34-42.

[93] Raeesi V, Chan WCW. Improving nanoparticle diffusion through tumor collagen matrix by photo-thermal gold nanorods. Nanoscale. 2016;8:12524-12530.

[94] Piehler S, Wucherpfennig L, Tansi FL, et al. Hyperthermia affects collagen fiber architecture and induces apoptosis in pancreatic and fibroblast tumor hetero-spheroids in vitro. Nanomedicine Nanotechnol Biol Med. 2020;102183. 
[95] Kolosnjaj-Tabi J, Kralj S, Griseti E, et al. Magnetic Silica-Coated Iron Oxide Nanochains as Photothermal Agents, Disrupting the Extracellular Matrix, and Eradicating Cancer Cells. Cancers. 2019;11:2040.

[96] Ta T, Bartolak-Suki E, Park E-J, et al. Localized delivery of doxorubicin in vivo from polymermodified thermosensitive liposomes with MR-guided focused ultrasound-mediated heating. J Controlled Release. 2014;194:71-81.

[97] Beola L, Asín L, Fratila RM, et al. Dual Role of Magnetic Nanoparticles as Intracellular Hotspots and Extracellular Matrix Disruptors Triggered by Magnetic Hyperthermia in 3D Cell Culture Models. ACS Appl Mater Interfaces. 2018;10:44301-44313.

[98] Li J, Xie C, Huang J, et al. Semiconducting Polymer Nanoenzymes with Photothermic Activity for Enhanced Cancer Therapy. Angew Chem Int Ed. 2018;57:3995-3998.

[99] Ramos-Docampo MA, Fernández-Medina M, Taipaleenmäki E, et al. Microswimmers with Heat Delivery Capacity for 3D Cell Spheroid Penetration. ACS Nano. 2019;13:12192-12205.

[100] Sebeke L, Deenen DA, Maljaars E, et al. Model predictive control for MR-HIFU-mediated, uniform hyperthermia. Int J Hyperthermia. 2019;36:1039-1049.

[101] Hancock HA, Smith LH, Cuesta J, et al. Investigations into Pulsed High-Intensity Focused Ultrasound-Enhanced Delivery: Preliminary Evidence for a Novel Mechanism. Ultrasound Med Biol. 2009;35:1722-1736.

[102] Lee $\mathrm{S}$, Han $\mathrm{H}, \mathrm{Koo} \mathrm{H}$, et al. Extracellular matrix remodeling in vivo for enhancing tumortargeting efficiency of nanoparticle drug carriers using the pulsed high intensity focused ultrasound. J Controlled Release. 2017;263:68-78.

[103] Jiang T, Zhang B, Shen S, et al. Tumor Microenvironment Modulation by Cyclopamine Improved Photothermal Therapy of Biomimetic Gold Nanorods for Pancreatic Ductal Adenocarcinomas. ACS Appl Mater Interfaces. 2017;9:31497-31508.

[104] • - Tan T, Hu H, Wang H, et al. Bioinspired lipoproteins-mediated photothermia remodels tumor stroma to improve cancer cell accessibility of second nanoparticles. Nat Commun. 2019;10:3322.

A very elegant and efficient 2-step protocol based on hyperthermia that plays with remodelling of ECM before treatment by an anti-cancer drug

[105] Sorrin AJ, Ruhi MK, Ferlic NA, et al. Photodynamic Therapy and the Biophysics of the Tumor Microenvironment. Photochem Photobiol. 2020;96:232-259.

[106] Krammer B. Vascular effects of photodynamic therapy. Anticancer Res. 2001;21:4271-4277.

[107] Chen B, Pogue BW, Hoopes PJ, et al. Vascular and cellular targeting for photodynamic therapy. Crit Rev Eukaryot Gene Expr. 2006;16:279-305.

[108] Fingar VH, Wieman TJ, Wiehle SA, et al. The role of microvascular damage in photodynamic therapy: the effect of treatment on vessel constriction, permeability, and leukocyte adhesion. Cancer Res. 1992;52:4914-4921.

[109] Fingar VH, Wieman TJ, Doak KW. Changes in Tumor Interstitial Pressure Induced by Photodynamic Therapy. Photochem Photobiol. 1991;53:763-768. 
[110] Malik Z, Faraggi A, Savion N. Ultrastructural damage in photosensitized endothelial cells: Dependence on hematoporphyrin delivery pathways. J Photochem Photobiol B. 1992;14:359-368.

[111] Leunig A, Staub F, Plesnila N, et al. Effect of photodynamic treatment of human endothelial cells on cell volume and cell viability. Int J Oncol. 1996;8:1217-1221.

[112] Sporn LA, Foster TH. Photofrin and Light Induces Microtubule Depolymerization in Cultured Human Endothelial Cells. Cancer Res. 1992;52:3443-3448.

[113] Gil M, Bieniasz M, Seshadri M, et al. Photodynamic therapy augments the efficacy of oncolytic vaccinia virus against primary and metastatic tumours in mice. $\mathrm{Br} \mathrm{J}$ Cancer. 2011;105:1512-1521.

[114] Snyder JW, Greco WR, Bellnier DA, et al. Photodynamic therapy: a means to enhanced drug delivery to tumors. Cancer Res. 2003;63:8126-8131.

[115] Cheng C, Debefve E, Haouala A, et al. Photodynamic therapy selectively enhances liposomal doxorubicin uptake in sarcoma tumors to rodent lungs. Lasers Surg Med. 2010;42:391-399.

[116] Perentes JY, Wang $Y$, Wang $X$, et al. Low-dose vascular photodynamic therapy decreases tumor interstitial fluid pressure, which promotes liposomal doxorubicin distribution in a murine sarcoma metastasis model. Transl Oncol. 2014;7:393-399.

[117] Sano K, Nakajima T, Choyke PL, et al. The Effect of Photoimmunotherapy Followed by Liposomal Daunorubicin in a Mixed Tumor Model: A Demonstration of the Super-Enhanced Permeability and Retention Effect after Photoimmunotherapy. Mol Cancer Ther. 2014;13:426-432.

[118] Luo D, Carter KA, Miranda D, et al. Chemophototherapy: an emerging treatment option for solid tumors. Adv Sci. 2017;4:1600106.

[119] Lv T, Huang Z-F, Wang H-W, et al. Evaluation of collagen alteration after topical photodynamic therapy (PDT) using second harmonic generation (SHG) microscopy - in vivo study in a mouse model. Photodiagnosis Photodyn Ther. 2012;9:164-169.

[120] Mills S j., Farrar M d., Ashcroft G s., et al. Topical photodynamic therapy following excisional wounding of human skin increases production of transforming growth factor- $\beta 3$ and matrix metalloproteinases 1 and 9 , with associated improvement in dermal matrix organization. $\mathrm{Br} \mathrm{J}$ Dermatol. 2014;171:55-62.

[121] Ryu A-R, Lee M-Y. Chlorin e6-mediated photodynamic therapy promotes collagen production and suppresses MMPs expression via modulating AP-1 signaling in P. acnes-stimulated $\mathrm{HaCaT}$ cells. Photodiagnosis Photodyn Ther. 2017;20:71-77.

[122] Yova DM, Hovhannisyan VA, Theodossiou T. Photochemical effects and hypericin photosensitized processes in collagen. J Biomed Opt. 2001;6:52-58.

[123] Hovhannisyan V, Guo HW, Hovhannisyan A, et al. Photo-induced processes in collagenhypericin system revealed by fluorescence spectroscopy and multiphoton microscopy. Biomed Opt Express. 2014;5:1355-1362. 
[124] Hovhannisyan V, Hovhannisyan A, Ghukasyan V, et al. Hypericin-mediated selective photomodification of connective tissues. Appl Phys Lett. 2014;105:263701.

[125] Ferrario A, Chantrain CF, von Tiehl K, et al. The matrix metalloproteinase inhibitor prinomastat enhances photodynamic therapy responsiveness in a mouse tumor model. Cancer Res. 2004;64:2328-2332.

[126] Karrer S, Bosserhoff AK, Weiderer P, et al. Influence of 5-aminolevulinic acid and red light on collagen metabolism of human dermal fibroblasts. J Invest Dermatol. 2003;120:325-331.

[127] Zhang C, Wang J, Chou A, et al. Photodynamic therapy induces antifibrotic alterations in primary human vocal fold fibroblasts. The Laryngoscope. 2018;128:E323-E331.

[128] Biel MA. Photodynamic Therapy Treatment of Early Oral and Laryngeal Cancers. Photochem Photobiol. 2007;83:1063-1068.

[129] Ihsanullah KM, Kumar BN, Zhao Y, et al. Stepwise-activatable hypoxia triggered nanocarrierbased photodynamic therapy for effective synergistic bioreductive chemotherapy. Biomaterials. 2020;245:119982.

[130] Tachibana K, Uchida T, Ogawa K, et al. Induction of cell-membrane porosity by ultrasound. The Lancet. 1999;353:1409.

[131] Frenkel V. Ultrasound mediated delivery of drugs and genes to solid tumors. Adv Drug Deliv Rev. 2008;60:1193-1208.

[132] Ferrara K, Pollard R, Borden M. Ultrasound Microbubble Contrast Agents: Fundamentals and Application to Gene and Drug Delivery. Annu Rev Biomed Eng. 2007;9:415-447.

[133] Hernot S, Klibanov AL. Microbubbles in ultrasound-triggered drug and gene delivery. Adv Drug Deliv Rev. 2008;60:1153-1166.

[134] Lentacker I, De Cock I, Deckers R, et al. Understanding ultrasound induced sonoporation: Definitions and underlying mechanisms. Adv Drug Deliv Rev. 2014;72:49-64.

[135] Tharkar P, Varanasi R, Wong WSF, et al. Nano-Enhanced Drug Delivery and Therapeutic Ultrasound for Cancer Treatment and Beyond. Front Bioeng Biotechnol. 2019;7:324.

[136] Skyba DM, Price RJ, Linka AZ, et al. Direct in vivo visualization of intravascular destruction of microbubbles by ultrasound and its local effects on tissue. Circulation. 1998;98:290-293.

[137] Chen H, Kreider W, Brayman AA, et al. Blood Vessel Deformations on Microsecond Time Scales by Ultrasonic Cavitation. Phys Rev Lett. 2011;106:034301.

[138] Chen H, Brayman AA, Evan AP, et al. Preliminary observations on the spatial correlation between short-burst microbubble oscillations and vascular bioeffects. Ultrasound Med Biol. 2012;38:2151-2162.

[139] Wamel A van, Kooiman K, Emmer M, et al. Ultrasound microbubble induced endothelial cell permeability. J Controlled Release. 2006;116:e100-e102. 
[140] Patil AV, Rychak JJ, Klibanov AL, et al. Real-Time Technique for Improving Molecular Imaging and Guiding Drug Delivery in Large Blood Vessels: In Vitro and Ex Vivo Results. Mol Imaging. 2011;10:7290.2011.00002.

[141] Meijering BDM, Henning RH, Gilst WHV, et al. Optimization of ultrasound and microbubbles targeted gene delivery to cultured primary endothelial cells. J Drug Target. 2007;15:664-671.

[142] Juffermans LM, Meijering BDM, Henning RH, et al. Ultrasound and Microbubble-Targeted Delivery of Small Interfering RNA Into Primary Endothelial Cells Is More Effective Than Delivery of Plasmid DNA. Ultrasound Med Biol. 2014;40:532-540.

[143] Meijering BDM, Juffermans LJM, van Wamel A, et al. Ultrasound and Microbubble-Targeted Delivery of Macromolecules Is Regulated by Induction of Endocytosis and Pore Formation. Circ Res. 2009;104:679-687.

[144] Juffermans LMM, van Dijk A, Jongenelen CAM, et al. Ultrasound and Microbubble-Induced Intra- and Intercellular Bioeffects in Primary Endothelial Cells. Ultrasound Med Biol. 2009;35:1917-1927.

[145] Bekeredjian R, Kroll RD, Fein E, et al. Ultrasound Targeted Microbubble Destruction Increases Capillary Permeability in Hepatomas. Ultrasound Med Biol. 2007;33:1592-1598.

[146] Mukherjee D, Wong J, Griffin B, et al. Ten-fold augmentation of endothelial uptake of vascular endothelial growth factor with ultrasound after systemic administration. J Am Coll Cardiol. 2000;35:1678-1686.

[147] Cochran MC, Eisenbrey JR, Soulen MC, et al. Disposition of ultrasound sensitive polymeric drug carrier in a rat hepatocellular carcinoma model. Acad Radiol. 2011;18:1341-1348.

[148] Yan F, Li L, Deng Z, et al. Paclitaxel-liposome-microbubble complexes as ultrasound-triggered therapeutic drug delivery carriers. J Controlled Release. 2013;166:246-255.

[149] Qin J, Wang T-Y, Willmann JK. Sonoporation: applications for cancer therapy. Ther Ultrasound. Springer; 2016. p. 263-291.

[150] Chowdhury SM, Wang T-Y, Bachawal S, et al. Ultrasound-guided therapeutic modulation of hepatocellular carcinoma using complementary microRNAs. J Controlled Release. 2016;238:272-280.

[151] Devulapally R, Sekar NM, Sekar TV, et al. Polymer nanoparticles mediated codelivery of antimiR-10b and antimiR-21 for achieving triple negative breast cancer therapy. ACS Nano. 2015;9:2290-2302.

[152] - Han H, Kim D, Jang $Y$, et al. Focused ultrasound-triggered chemo-gene therapy with multifunctional nanocomplex for enhancing therapeutic efficacy. J Controlled Release. 2020;322:346-356.

Focused ultrasound-responsive nanocomplex can be regarded as a novel drug delivery system using sonoporation effect

[153] - Kolosnjaj-Tabi J, Gibot L, Fourquaux I, et al. Electric field-responsive nanoparticles and electric fields: physical, chemical, biological mechanisms and therapeutic prospects. Adv Drug Deliv Rev. 2019;138:56-67. 
Focused review on electroporation and electric field-responsive nanoparticles for biomedical purposes

[154] Yarmush ML, Golberg A, Serša G, et al. Electroporation-Based Technologies for Medicine: Principles, Applications, and Challenges. Annu Rev Biomed Eng. 2014;16:295-320.

[155] Gehl J, Sersa G, Matthiessen LW, et al. Updated standard operating procedures for electrochemotherapy of cutaneous tumours and skin metastases. Acta Oncol. 2018;57:874882.

[156] Ramirez L, Orlowski S, An D, et al. Electrochemotherapy on liver tumours in rabbits. Br J Cancer. 1998;77:2104-2111.

[157] Sersa G, Beravs K, Cemazar M, et al. Contrast enhanced MRI assessment of tumor blood volume after application of electric pulses. Electro- Magnetobiology. 1998;17:299-306.

[158] Gehl J, Skovsgaard T, Mir LM. Vascular reactions to in vivo electroporation: characterization and consequences for drug and gene delivery. Biochim Biophys Acta BBA - Gen Subj. 2002;1569:51-58.

[159] Sersa G, Jarm T, Kotnik T, et al. Vascular disrupting action of electroporation and electrochemotherapy with bleomycin in murine sarcoma. Br J Cancer. 2008;98:388-398.

[160] Gehl J, Geertsen PF. Efficient palliation of haemorrhaging malignant melanoma skin metastases by electrochemotherapy. Melanoma Res. 2000;10:585-589.

[161] Markelc B, Bellard E, Sersa G, et al. In Vivo Molecular Imaging and Histological Analysis of Changes Induced by Electric Pulses Used for Plasmid DNA Electrotransfer to the Skin: A Study in a Dorsal Window Chamber in Mice. J Membr Biol. 2012;245:545-554.

[162] Markelc B, Bellard E, Sersa G, et al. Increased permeability of blood vessels after reversible electroporation is facilitated by alterations in endothelial cell-to-cell junctions. J Controlled Release. 2018;276:30-41.

[163] Sersa G, Cemazar M, Miklavcic D, et al. Tumor blood flow modifying effect of electrochemotherapy with bleomycin. Anticancer Res. 1999;19:4017-4022.

[164] Sersa G, Cemazar M, Parkins CS, et al. Tumour blood flow changes induced by application of electric pulses. Eur J Cancer. 1999;35:672-677.

[165] Sersa G, Krzic M, Sentjurc M, et al. Reduced blood flow and oxygenation in SA-1 tumours after electrochemotherapy with cisplatin. Br J Cancer. 2002;87:1047-1054.

[166] Kanthou C, Kranjc S, Sersa G, et al. The endothelial cytoskeleton as a target of electroporation-based therapies. Mol Cancer Ther. 2006;5:3145-3152.

[167] Meulenberg CJW, Todorovic V, Cemazar M. Differential cellular effects of electroporation and electrochemotherapy in monolayers of human microvascular endothelial cells. PloS One. 2012; 7:e52713.

[168] Kodama H, Shamay Y, Kimura Y, et al. Electroporation-induced changes in tumor vasculature and microenvironment can promote the delivery and increase the efficacy of sorafenib nanoparticles. Bioelectrochemistry Amst Neth. 2019;130:107328. 
[169] Kis E, Baltás E, Kinyó Á, et al. Successful Treatment of Multiple Basaliomas with Bleomycinbased Electrochemotherapy: A Case Series of Three Patients with Gorlin-Goltz Syndrome. Acta Derm Venereol. 2012;92:648-651.

[170] Campana LG, Mocellin S, Basso M, et al. Bleomycin-Based Electrochemotherapy: Clinical Outcome from a Single Institution's Experience with 52 Patients. Ann Surg Oncol. 2009;16:191-199.

[171] Maor E, Ivorra A, Leor J, et al. The effect of irreversible electroporation on blood vessels. Technol Cancer Res Treat. 2007;6:307-312.

[172] Golberg A, Villiger M, Broelsch GF, et al. Skin regeneration with all accessory organs following ablation with irreversible electroporation. J Tissue Eng Regen Med. 2018;12:98113.

[173] Weltmann KD, Von Woedtke T. Plasma medicine-current state of research and medical application. Plasma Phys Control Fusion. 2016;59:014031.

[174] Privat-Maldonado A, Bengtson C, Razzokov J, et al. Modifying the Tumour Microenvironment: Challenges and Future Perspectives for Anticancer Plasma Treatments. Cancers. 2019;11:1920.

[175] Arndt S, Unger P, Berneburg M, et al. Cold atmospheric plasma (CAP) activates angiogenesisrelated molecules in skin keratinocytes, fibroblasts and endothelial cells and improves wound angiogenesis in an autocrine and paracrine mode. J Dermatol Sci. 2018;89:181-190.

[176] Duchesne C, Banzet S, Lataillade J-J, et al. Cold atmospheric plasma modulates endothelial nitric oxide synthase signalling and enhances burn wound neovascularisation. J Pathol. 2019;249:368-380.

[177] Nikitovic D, Corsini E, Kouretas D, et al. ROS-major mediators of extracellular matrix remodeling during tumor progression. Food Chem Toxicol. 2013;61:178-186.

[178] Keyvani A, Atyabi SM, Sardari S, et al. Effects of cold atmospheric plasma jet on collagen structure in different treatment times. Basic Res J Med Clin Sci. 2017;6:84-90.

[179] Dong X, Chen M, Wang Y, et al. A Mechanistic study of Plasma Treatment Effects on Demineralized Dentin Surfaces for Improved Adhesive/Dentin Interface Bonding. Clin Plasma Med. 2014;2:11-16.

[180] Samouillan V, Merbahi N, Yousfi M, et al. Effect of Low-Temperature Plasma Jet on Thermal Stability and Physical Structure of Type I Collagen. IEEE Trans Plasma Sci. 2012;40:16881695.

[181] Wiegand C, Fink S, Beier O, et al. Dose- and Time-Dependent Cellular Effects of Cold Atmospheric Pressure Plasma Evaluated in 3D Skin Models. Skin Pharmacol Physiol. 2016;29:257-265.

[182] Arndt S, Unger P, Wacker E, et al. Cold atmospheric plasma (CAP) changes gene expression of key molecules of the wound healing machinery and improves wound healing in vitro and in vivo. PloS One. 2013;8:e79325-e79325. 
[183] Shi X, Cai J, Xu G, et al. Effect of Cold Plasma on Cell Viability and Collagen Synthesis in Cultured Murine Fibroblasts. Plasma Sci Technol. 2016;18:353-359.

[184] Vijayarangan V, Delalande A, Dozias S, et al. Cold Atmospheric Plasma Parameters Investigation for Efficient Drug Delivery in HeLa Cells. IEEE Trans Radiat Plasma Med Sci. 2018;2:109-115.

[185] Jinno M, Tachibana K, Motomura H, et al. Improvement of efficiency and viability in plasma gene transfection by plasma minimization and optimization electrode configuration. Jpn J Appl Phys. 2016;55:07LG09.

[186] Zhu W, Lee S-J, Castro NJ, et al. Synergistic Effect of Cold Atmospheric Plasma and Drug Loaded Core-shell Nanoparticles on Inhibiting Breast Cancer Cell Growth. Sci Rep. 2016;6:21974.

[187] Kim GC, Kim GJ, Park SR, et al. Air plasma coupled with antibody-conjugated nanoparticles: a new weapon against cancer. J Phys Appl Phys. 2008;42:032005.

[188] Wang M, Geilich BM, Keidar M, et al. Killing malignant melanoma cells with protoporphyrin IX-loaded polymersome-mediated photodynamic therapy and cold atmospheric plasma. Int J Nanomedicine. 2017;12:4117-4127.

[189] - Raavé R, van Kuppevelt TH, Daamen WF. Chemotherapeutic drug delivery by tumoral extracellular matrix targeting. J Controlled Release. 2018;274:1-8.

Description of the emerging field of drug delivery targeting tumour ECM in order to affect all subsets of cells within the tumour microenvironment.

[190] Guo J, Zeng H, Chen Y. Emerging Nano Drug Delivery Systems Targeting Cancer-Associated Fibroblasts for Improved Antitumor Effect and Tumor Drug Penetration. Mol Pharm. 2020;17:1028-1048.

[191] Van der Steen SC, Raavé R, Langerak S, et al. Targeting the extracellular matrix of ovarian cancer using functionalized, drug loaded lyophilisomes. Eur J Pharm Biopharm. 2017;113:229-239.

[192] Li L, Zhou S, Lv N, et al. Photosensitizer-Encapsulated Ferritins Mediate Photodynamic Therapy against Cancer-Associated Fibroblasts and Improve Tumor Accumulation of Nanoparticles. Mol Pharm. 2018;15:3595-3599.

[193] Vail DM, Amantea MA, Colbern GT, et al. Pegylated liposomal doxorubicin: proof of principle using preclinical animal models and pharmacokinetic studies. Semin Oncol. Elsevier; 2004. p. 16-35.

[194] Dreher MR, Liu W, Michelich CR, et al. Tumor vascular permeability, accumulation, and penetration of macromolecular drug carriers. J Natl Cancer Inst. 2006;98:335-344.

[195] Wang $\mathrm{H}$, Lin $\mathrm{Y}$, Nienhaus $\mathrm{K}$, et al. The protein corona on nanoparticles as viewed from a nanoparticle-sizing perspective. WIREs Nanomedicine Nanobiotechnology. 2018;10:e1500.

[196] Cai R, Chen C. The Crown and the Scepter: Roles of the Protein Corona in Nanomedicine. Adv Mater Deerfield Beach Fla. 2019;31:e1805740. 
[197] • Appelboom G, Detappe A, LoPresti M, et al. Stereotactic modulation of blood-brain barrier permeability to enhance drug delivery. Neuro-Oncol. 2016;18:1601-1609.

3 Review on physical ways to disrupt blood-brain barrier to enhance drug delivery

4 [198] Hynynen K. Focused ultrasound for blood-brain disruption and delivery of therapeutic molecules into the brain. Expert Opin Drug Deliv. 2007;4:27-35.

[199] Lorenzo MF, Thomas SC, Kani Y, et al. Temporal Characterization of Blood-Brain Barrier Disruption with High-Frequency Electroporation. Cancers. 2019;11:1850.

8 